\title{
Wilson Loops and QCD/String Scattering Amplitudes
}

\author{
Yuri Makeenko* \\ Institute of Theoretical and Experimental Physics \\ 117218 Moscow, Russia \\ makeenko@itep.ru \\ and \\ Poul Olesen* \\ The Niels Bohr International Academy \\ The Niels Bohr Institute \\ Blegdamsvej 17, 2100 Copenhagen Ø, Denmark \\ polesen@nbi.dk
}

\begin{abstract}
We generalize modern ideas about the duality between Wilson loops and scattering amplitudes in $\mathcal{N}=4 \mathrm{SYM}$ to large $N$ QCD by deriving a general relation between QCD meson scattering amplitudes and Wilson loops. We then investigate properties of the open-string disk amplitude integrated over reparametrizations. When the Wilson loop is approximated by the area behavior, we find that the QCD scattering amplitude is a convolution of the standard Koba-Nielsen integrand and a kernel. As usual poles originate from the first factor, whereas no (momentum dependent) poles can arise from the kernel. We show that the kernel becomes a constant when the number of external particles becomes large. The usual Veneziano amplitude then emerges in the kinematical regime where the Wilson loop can be reliably approximated by the area behavior. In this case we obtain a direct duality between Wilson loops and scattering amplitudes when spatial variables and momenta are interchanged, in analogy with the $\mathcal{N}=4$ SYM case.
\end{abstract}

*Also at the Institute for Advanced Cycling, Blegdamsvej 19, 2100 Copenhagen Ø, Denmark 


\section{Introduction}

The relation between planar diagrams and dual resonance models has a long history since the pioneering works [1]. A long-standing belief [2] is that $S U(N)$ Yang-Mills theory is equivalent at large $N$ to a free string, while the $1 / N$-expansion corresponds to interactions of the string. ${ }^{1}$ A great recent progress along this line is associated for $\mathcal{N}=4$ super YangMills theory (SYM) with the AdS/CFT correspondence [4, 5] (see Ref. [6] for a review), where the strong-coupling limit of SYM is described by supergravity in anti-de Sitter space $A d S_{5} \times S^{5}$.

The (finite part of the) 4-gluon Maximally-Helicity-Violating on-shell scattering amplitude in SYM theory has the form

$$
A(s, t)=A_{\text {tree }} \mathrm{e}^{f(\lambda) \log ^{2}(s / t)}
$$

(where $s$ and $t$ are usual Mandelstam's variables) as was conjectured [7] on the basis of three-loop calculations. To explain Eq. (1.1), the Wilson-loop/scattering-amplitude (WL/SA) duality was introduced [8] at large 't Hooft couplings $\lambda$, which has been then advocated in SYM perturbation theory [9]. This duality (for a review see Ref. [10]) states that the scattering amplitude (divided by the kinematical factor $A_{\text {tree }}$ ) equals the Wilson loop for a polygon whose vertices $x_{i}$ are related to the momenta $p_{i}$ of scattering gluons by

$$
p_{i}=K\left(x_{i}-x_{i-1}\right),
$$

where $K=1 / 2 \pi \alpha^{\prime}$ is the string tension.

Our goal in this paper is to find out what features of the described WL/SA duality (if any) remain valid for QCD and, in particular, how is it possible to maintain the relation of the type (1.2) which would relate large momenta in scattering amplitudes with loops of large size. Of course this is not possible in QCD perturbation theory, where $|p| \sim 1 /|x|$ because of dimensional ground. But nonperturbatively a dimensional parameter $K \approx(400 \mathrm{MeV})^{2}$ appears in $\mathrm{QCD}$, which shows up in the area-law behavior of asymptotically large Wilson loops:

$$
W(C) \stackrel{\text { large } C}{\propto} \mathrm{e}^{-K S_{\min }(C)},
$$

where $S_{\min }(C)$ is the area of the minimal surface bounded by $C$, that results in confinement. Strictly speaking, this requires the limit of the large number of colors $N$ or the quenched approximation.

As is well-known by now, a string theory, which QCD is supposedly equivalent to, is not the simplest Nambu-Goto string. Some extra degrees of freedom living on the string are required which are most probably conveniently described by a presence of extra dimensions. The asymptotic behavior (1.3) is nevertheless universal for large loops.

In this paper the main topic is an investigation of the relation between the Wilson loop and the corresponding amplitude in large $N$ QCD, or, alternatively, quenched QCD

\footnotetext{
${ }^{1}$ See e.g. Ref. [3] for an introduction and review of the old works on the QCD/string correspondence.
} 
for any $N$. In general this involves integration over an infinite number of loops. However, if one considers large loops there is a considerable amount of evidence from lattice gauge calculations in $2+1$ and $3+1$ dimensions for various $N$ 's that the Nambu-Goto action describes the behavior of the Wilson loops quite well. To give an example, in [11] it is shown with unprecedented precision that the static quark potential in quenched $S U(3)$ lattice gauge theory is well described by the first two terms $(\propto r$ and the Lüscher term $\propto 1 / r)$ in a long-distance expansion of the Nambu-Goto action. Furthermore, in both three and four dimensions the transition from perturbative to string behavior takes place "at surprisingly small distances" [11]. There also exists a number of other comparisons between results from the Nambu-Goto action, e.g. the closed string spectrum and $S U(N)$ for various values of $N$, see [12] where further references can be found.

The various results from lattice gauge theories can be summarized by the statement that the Nambu-Goto action describes the large and not so large [11] distance behavior of quenched QCD surprisingly well. This action has the well known anomaly for $d \neq 26$, which however is suppressed for long strings [13]. The remarkable success of the NambuGoto string as an effective action led us to reconsider the relation between the Wilson loop $W(C)$ and the corresponding amplitude. The idea is to perform the unpleasant sum over all $C$ 's by inserting the Nambu-Goto action in $W(C)$. Then the sum over $C$ becomes an integration over the string field $x^{\mu}(\sigma, \tau)$, and is at least in principle controllable. In practice, in this paper we start out with a more modest program, where only the area behavior of $W(C)$ is inserted in the form of the disk amplitude. Thus, the effects of the Lüscher term is not included in the investigation reported in this paper.

The present paper is an extended version of Ref. [14], but some of the reported results are novel. In Sect. 2 we derive a relation between the $M$-meson scattering amplitude and the Wilson loop for the case of fermion and scalar quarks, valid for QCD in the large $N$ limit. Our results are general in the sense that if the Wilson loop is known one can obtain the scattering amplitudes by performing some path integrations. In Sect. 3 we then take up the old idea that the large $N$ QCD Wilson loop should be identified with the disk amplitude in certain string models as far as the leading large distance behavior is concerned. We emphasize that it is crucial in this construction to integrate the string disk amplitude over reparametrizations of the boundary contour. In Sect. 4 we show, taking a functional Fourier transform to momentum space, how the disk amplitude leads to the Koba-Nielsen amplitude. Integrating over the reparametrizations, we also derive projective-invariant off-shell scattering amplitudes.

In Sect. 5 we return to the general formula from Sect. 2 for the relation between the meson (made from fermion quarks) scattering amplitude and the Wilson loop. Here we insert the area law (with no subleading perimeter term, Lüscher term, ...) and for the (off-shell) $M$-particle amplitude we derive the formula

$$
G\left(\Delta p_{1}, \ldots, \Delta p_{M}\right) \propto \prod_{1}^{M-1} \int_{0}^{\phi_{i+1}} \mathrm{~d} \phi_{i} \prod_{j=1}^{M}\left[\frac{\sin \left[\left(\phi_{j+1}-\phi_{j}\right) / 2\right] \sin \left[\left(\phi_{j}-\phi_{j-1}\right) / 2\right]}{\sin \left[\left(\phi_{j+1}-\phi_{j-1}\right) / 2\right] \sin ^{2}\left(\phi_{j} / 2\right)}\right]^{\Delta p_{j}^{2} / 4 \pi K}
$$




$$
\begin{gathered}
\times \exp \left(\frac{1}{4 \pi K} \sum_{\substack{i, j=1 \\
i \neq j}}^{M} \Delta p_{i} \Delta p_{j} \ln \left(1-\cos \left(\phi_{i}-\phi_{j}\right)\right)\right) \mathcal{K}\left(\phi_{1}, \ldots, \phi_{M-1} ; \Delta p_{1}, \ldots, \Delta p_{M}\right), \\
\phi_{0}=0, \quad \phi_{M}=2 \pi,
\end{gathered}
$$

where the $\Delta p$ 's are particle momenta and where the "kernel" $\mathcal{K}$ in general has no momentum dependent singularities for $\phi_{i} \rightarrow \phi_{j}$. The first factor in the integrand on the right-hand side is the well-known Koba-Nielsen integrand, and it produces poles in the integral for $\phi_{i} \rightarrow \phi_{j}$. Physically these poles only occur when the area law is a good representation of the Wilson loop, which means that the area should be large and correspondingly the momenta should also be large. Thus, due to this condition there is no tachyon (or other low lying states) in the spectrum, as one would indeed expect in QCD. We further show that in the case of large $M$ the kernel $\mathcal{K}$ degenerates to a factor which is essentially independent of the $\Delta p$ 's. This implies that when the area behavior of the Wilson loop dominates the dynamics we get the interesting result that when many particles are produced in some collision then the scattering is given by a Veneziano type of amplitude. Of course, this is for large $N$ QCD, but it would be interesting to see to which extent this would be valid at the LHC collider with $N=3$.

The condition that the various momentum transfers should be large in order that the area behavior for the Wilson loop can be inserted is only a necessary but not sufficient condition for the validity of our approach. This is important in view of one of the historical reasons for not using the Veneziano amplitude in strong interaction phenomenology: at large transverse momenta this amplitude decreases exponentially in contrast to the experimental data. If we consider the 4-point function in terms of the usual Mandelstam variables $s$ and $t$, our approach is valid when $1 / \alpha^{\prime} \lesssim-t \ll s$ in Minkowski space, because it dominates over other contributions. However, if $-t \sim s$, this is no longer true, because then the Veneziano amplitude becomes a tiny exponentially decreasing function and other contributions lead to a power decrease of the amplitude, which of course is far more important than an exponential decrease. Therefore the area behaved Wilson loop no longer dominates when $-t \sim s$.

In Sect. 6 we find a relation between the space variables and the external momenta which is the QCD analogue of the SYM WL/SA duality exhibited in Eq. (1.2). If $z(\phi)$ describes the contour of the Wilson loop as a function of an angular parametrization $\phi$, then

$$
z(\phi)=\frac{1}{K} \sum_{i=1}^{M} p_{i} \Theta\left(\phi-\phi_{i}\right) \Theta\left(\phi-\phi_{i+1}\right),
$$

where $p_{i}$ are related to the external momenta $\Delta p_{i}$ by $\Delta p_{i}=p_{i-1}-p_{i}$, and $\Theta$ is the usual step function. If this expression is inserted in the $z$-dependent Wilson loop, it reproduces the scattering amplitude with a large number $M$ of external particles when the $\phi_{i}$ 's are integrated over. Thus, the relevant contours are given by the constant momentum vectors, in complete analogy with Eq. (1.2). The difference with the supersymmetric case is that in QCD we have to integrate over the parameters $\phi_{i}$ which represent the points in parameter 
space where the momenta enter. Otherwise (1.5) is like Eq. (1.2), since $z(\phi)$ equals $p_{i} / K$ in the interval from the point $x_{i}$ to $x_{i+1}$, so the vector $x_{i+1}-x_{i}$ equals $\Delta p_{i} / K$, as in Eq. (1.2).

Some more technical details are discussed in the Appendices. In Appendix A we give an example of how to operate with path integrals related to the ordering of gamma matrices. In Appendix B we review the modern approach to the minimal area as a boundary functional. To illustrate the asymptotic area behavior, we evaluate in Appendix $\mathrm{C}$ the integral over reparametrizations in the disk amplitude for a large circle. Appendix D is devoted to calculations of the path integral over reparametrizations. In Appendix E we consider a scattering amplitude which appears in QCD, when the Wilson loop is substituted by an exact area law, i.e. Eq. (1.3) holds not only asymptotically but for all contours.

\section{QCD amplitudes dual to Wilson loops: general re- sults}

In large $N$ QCD, Green's functions of $M$ colorless composite quark operators (e.g. $\left.\bar{q}\left(x_{i}\right) q\left(x_{i}\right)\right)$ are given by the sum over all Wilson loops passing via the points $x_{i}(i=1, \ldots, M)$, where the operators are inserted. This approach was first advocated on the lattice [15] and then extended [16] to the continuum. To obtain a scattering amplitude, one makes the Fourier transformation with respect to $x_{i}$ and takes the corresponding momenta $p_{i}$ on shell.

The weight for the summation over paths depends on both the quark spin and the quantum numbers of the operators (see Refs. [17, 3] for more detail). The simplest results are for fermion quarks and the scalar operators $\bar{q}\left(x_{i}\right) q\left(x_{i}\right)$ when the connected Green's function is

$$
G\left(x_{1}, \ldots, x_{M}\right) \equiv\left\langle\prod_{i=1}^{M} \bar{q}\left(x_{i}\right) q\left(x_{i}\right)\right\rangle_{\mathrm{conn}}=\sum_{C \ni} W(C) .
$$

Here $W(C)$ is the Wilson loop in pure Yang-Mills theory at large $N$. For finite $N$, correlators of several Wilson loops have to be taken into account.

In Euclidean space the standard weight for the summation over paths in Eq. (2.1) reads explicitly

$$
\begin{aligned}
& \left\langle\prod_{i=1}^{M} \bar{q}\left(x_{i}\right) q\left(x_{i}\right)\right\rangle=\prod_{\text {conn }}^{M} \int_{i=1}^{\infty} \mathrm{d} \tau_{i} \mathrm{e}^{-m \tau_{i}} \\
& \quad \times \int_{\substack{z_{i}(0)=x_{i-1} \\
z_{i}\left(\tau_{i}\right)=x_{i}}} \mathcal{D} z_{i}(t) \int \mathcal{D} k(t) \mathrm{sp} \mathrm{P} \exp \left(\mathrm{i} \int_{0}^{\tau_{i}} \mathrm{~d} t\left[\dot{z}_{i}(t) k(t)-\gamma(t) k(t)\right]\right) W(C),
\end{aligned}
$$

where the $i$-segment of the loop $C$ from $x_{i-1}$ to $x_{i}$ is represented by the function $z_{i}(t)$ $\left(0<t<\tau_{i}\right)$ and $x_{M}=x_{0}$ (since the loops are closed). The integration over the variable 
gamma matrix $\gamma_{\mu}(t)$ is explained in Appendix 7. Above we have introduced the convention to be used in the rest of the paper that dot means derivative with respect to whatever argument a function has. Thus, for some function $f$ we have $\dot{f}(x)=\mathrm{d} f(x) / \mathrm{d} x$, whereas $\dot{f}(y)=\mathrm{d} f(y) / \mathrm{d} y$.

Equation (2.2) is essentially derived in [3], where further references can be found. For the readers convenience we repeat the essential steps. In QCD the quark fields can be integrated out and we have the Feynman disentangling ${ }^{2}$

$$
\begin{aligned}
& \left\langle y\left|\frac{1}{\gamma_{\mu} \nabla_{\mu}+m}\right| x\right\rangle=\int_{0}^{\infty} \mathrm{d} \tau\left\langle y\left|\mathrm{e}^{-\tau\left(\gamma_{\mu} \nabla_{\mu}+m\right)}\right| x\right\rangle \\
& \quad=\int_{0}^{\infty} \mathrm{d} \tau \mathrm{e}^{-m \tau} \int \mathcal{D} k_{\mu} \mathrm{sp} \mathrm{P} \mathrm{e}^{-\int_{0}^{\tau} \mathrm{d} t \gamma_{\mu}(t)\left(\mathrm{i} k_{\mu}(t)-\mathrm{i} A_{\mu}(t)\right)} \delta\left(k_{\mu}(t)+\mathrm{i} \partial_{\mu}(t)\right) \delta(x-y),
\end{aligned}
$$

where sp and $\mathrm{P}$ act on the gammas and color matrices. The functional delta function has the representation

$$
\delta\left(k_{\mu}(t)+\mathrm{i} \partial_{\mu}(t)\right) \delta(x-y)=\int \mathcal{D} v_{\mu} \mathrm{e}^{\mathrm{i} \int_{0}^{\tau} \mathrm{d} t v_{\mu}(t)\left(k_{\mu}(t)+\mathrm{i} \partial_{\mu}(t)\right)} \delta(x-y) .
$$

Here $v_{\mu}(t)$ has no restrictions. Then

$$
\delta\left(k_{\mu}(t)+\mathrm{i} \partial_{\mu}(t)\right) \delta(x-y)=\int \mathcal{D} v_{\mu} \mathrm{e}^{\mathrm{i} \int_{0}^{\tau} \mathrm{d} t v_{\mu}(t) k_{\mu}(t)} \delta\left(x+\int_{0}^{\tau} \mathrm{d} t v(t)-y\right) .
$$

With $z_{\mu}(t)=x_{\mu}+\int_{0}^{t} \mathrm{~d} t^{\prime} v_{\mu}\left(t^{\prime}\right)$ we get

$$
\delta\left(k_{\mu}(t)+\mathrm{i} \partial_{\mu}(t)\right) \delta(x-y)=\int_{\substack{z(0)=x \\ z(\tau)=y}} \mathcal{D} z_{\mu} \mathrm{e}^{\mathrm{i} \int_{0}^{\tau} \mathrm{d} t \dot{z}_{\mu}(t) k_{\mu}(t)}
$$

Introducing Eq. (2.6) in Eq. (2.3), we obtain

$$
\begin{aligned}
\int_{0}^{\infty} \mathrm{d} \tau & \left\langle y\left|\mathrm{e}^{-\tau\left(\gamma_{\mu} \nabla_{\mu}+m\right)}\right| x\right\rangle \\
& =\int_{0}^{\infty} \mathrm{d} \tau \mathrm{e}^{-m \tau} \int \mathcal{D} k_{\mu} \int_{\substack{z(0)=x \\
z(\tau)=y}} \mathcal{D} z_{\mu} \mathrm{sp} \mathrm{P} \mathrm{e}^{\mathrm{i} \int_{0}^{\tau} \mathrm{d} t\left[\dot{z}_{\mu} k_{\mu}-\gamma_{\mu} k_{\mu}+\gamma_{\mu} A_{\mu}\right]} .
\end{aligned}
$$

Shifting the integration by $k_{\mu} \rightarrow k_{\mu}+A_{\mu}$, we get

$$
\begin{aligned}
\int_{0}^{\infty} \mathrm{d} \tau & \left\langle y\left|\mathrm{e}^{-\tau\left(\gamma_{\mu} \nabla_{\mu}+m\right)}\right| x\right\rangle \\
& =\int_{0}^{\infty} \mathrm{d} \tau \mathrm{e}^{-m \tau} \int \mathcal{D} k_{\mu} \int_{\substack{z(0)=x \\
z(\tau)=y}} \mathcal{D} z_{\mu} \mathrm{sp} \mathrm{P} \mathrm{e}^{\mathrm{i} \int_{0}^{\tau} \mathrm{d} t\left[\dot{z}_{\mu} k_{\mu}-\gamma_{\mu} k_{\mu}+A_{\mu} \dot{z}_{\mu}\right]}
\end{aligned}
$$

\footnotetext{
${ }^{2}$ We use the notation of [3]. The states $|x\rangle$ etc. are eigenstates of $x$ and the standard Feynman disentangling is used in (2.3).
} 
This result leads immediately to Eq. (2.2) since the contour of $C$ is composed of pieces each of which can be represented as in Eq. (2.8).

Introducing new proper-time variables

$$
\mathcal{T}_{i}=\sum_{j=1}^{i} \tau_{j}, \quad \mathcal{T} \equiv \mathcal{T}_{M}
$$

we rewrite Eq. (2.2) in a time-ordered form:

$$
\begin{aligned}
& G\left(x_{1}, \ldots, x_{M}\right) \\
& \quad=\int_{0}^{\infty} \mathrm{d} \mathcal{T} \mathrm{e}^{-m \mathcal{T}} \prod_{i=1}^{M-1} \int_{0}^{\mathcal{T}_{i+1}} \mathrm{~d} \mathcal{T}_{i} \int_{\begin{array}{c}
z(0)=x_{0} \\
z(\mathcal{T})=x_{i} \\
z(\mathcal{T})=x_{M} \equiv x_{0}
\end{array}} \mathcal{D} z(t) \mathcal{D} k(t) \text { sp P } \mathrm{e}^{\mathrm{i} \int_{0}^{\mathcal{T}} \mathrm{d} t[\dot{z}(t) k(t)-\gamma(t) k(t)]} W[z(t)],
\end{aligned}
$$

where the loop is represented by a single function $z_{\mu}(t)(0<t<\mathcal{T})$ :

$$
C \ni x_{1}, \ldots, x_{M}=\left\{z_{\mu}(t), \quad z(0)=x_{0}, \ldots, z\left(\mathcal{T}_{i}\right)=x_{i}, \ldots, z(\mathcal{T})=x_{M} \equiv x_{0}\right\}
$$

describing a closed loop passing via the (ordered) set of points $x_{i}$. It is essential here that $\mathcal{T}_{i} \leq \mathcal{T}_{i+1}$

Finally, we introduce the angular-type variables

$$
\begin{aligned}
\phi & =2 \pi \frac{t}{\mathcal{T}} \quad(0<\phi \leq 2 \pi), \\
\phi_{i} & =2 \pi \frac{\mathcal{T}_{i}}{\mathcal{T}} \quad\left(0<\phi_{i} \leq \phi_{i+1} \leq 2 \pi\right), \\
\phi_{M} & \equiv 2 \pi
\end{aligned}
$$

so that

$$
\begin{aligned}
& G\left(x_{1}, \ldots, x_{M}\right)=\int_{0}^{\infty} \mathrm{d} \mathcal{T}\left(\frac{\mathcal{T}}{2 \pi}\right)^{M-1} \mathrm{e}^{-m \mathcal{T}} \\
& \quad \times \prod_{i=1}^{M-1} \int_{0}^{\phi_{i+1}} \mathrm{~d} \phi_{i} \int_{\substack{z(0)=z(2 \pi)=x_{0} \\
z\left(\phi_{i}\right)=x_{i}}} \mathcal{D} z(\phi) \int \mathcal{D} k(\phi) \mathrm{sp} \mathrm{P} \mathrm{e}^{\mathrm{i} \int_{0}^{2 \pi} \mathrm{d} \phi[\dot{z}(\phi) k(\phi)-\mathcal{T} \gamma(\phi) k(\phi) / 2 \pi]} W[z(\phi)] .
\end{aligned}
$$

The on-shell $M$-particle amplitude can be obtained from the Green function (2.13) by applying the standard LSZ reduction formula. When making the Fourier transformation, it is convenient to represent $M$ momenta of the (all incoming) particles by the differences

$$
\Delta p_{i}=p_{i-1}-p_{i}
$$


Then momentum conservation is automatic while an (infinite) volume $V$ is produced, say, by integration over $x_{0}$. We therefore define

$$
G\left(\Delta p_{1}, \ldots, \Delta p_{M}\right)=\frac{1}{V} \prod_{i=1}^{M} \int \mathrm{d}^{4} x_{i} \mathrm{e}^{\mathrm{i} \sum_{i} \Delta p_{i} x_{i}} G\left(x_{1}, \ldots, x_{M}\right) .
$$

We can further rewrite this formula, introducing a momentum-space loop $p_{\mu}(\phi)$ which is piecewise constant:

$$
p(\phi)=p_{i} \quad \text { for } \phi_{i}<\phi<\phi_{i+1} .
$$

Noting that

$$
\dot{\vec{p}}(\phi)=-\sum_{i} \Delta \vec{p}_{i} \delta\left(\phi-\phi_{i}\right), \quad \Delta \vec{p}_{i} \equiv \vec{p}_{i-1}-\vec{p}_{i}
$$

we write

$$
\sum_{i} \Delta p_{i} x_{i}=-\int_{0}^{2 \pi} \mathrm{d} \phi \dot{p}(\phi) \cdot z(\phi)=\int_{0}^{2 \pi} \mathrm{d} \phi p(\phi) \cdot \dot{z}(\phi)
$$

which is manifestly parametric-invariant.

Inserting (2.13) into Eq. (2.15) and noting that

$$
\prod_{i=1}^{M} \int \mathrm{d}^{4} x_{i} \int_{\substack{z(0)=x_{0} \\ z\left(\phi_{i}\right)=x_{i} \\ z(2 \pi)=x_{0}}} \mathcal{D} z(\phi) F(z(\phi))=\int_{z(0)=z(2 \pi)} \mathcal{D} z(\phi) F(z(\phi)),
$$

where $F$ is some functional, we obtain

$$
\begin{aligned}
& G\left(\Delta p_{1}, \ldots, \Delta p_{M}\right)=\int_{0}^{\infty} \mathrm{d} \mathcal{T}\left(\frac{\mathcal{T}}{2 \pi}\right)^{M-1} \mathrm{e}^{-m \mathcal{T}} \prod_{i=1}^{M-1} \int_{0}^{\phi_{i+1}} \mathrm{~d} \phi_{i} \int \mathcal{D} k(\phi) \\
& \quad \times \int_{z(0)=z(2 \pi)=0} \mathcal{D} z(\phi) \mathrm{sp} \mathrm{P} \mathrm{e}^{\mathrm{i} \int_{0}^{2 \pi} \mathrm{d} \phi[(k(\phi)+p(\phi)) \cdot \dot{z}(\phi)-\mathcal{T} \gamma(\phi) k(\phi) / 2 \pi]} W[z(\phi)],
\end{aligned}
$$

where $p(\phi)$ is piecewise constant as is given by Eq. (2.16). We do not integrate over $z(0)=z(2 \pi)$ which would produce the (infinite) volume factor because of translational invariance.

In the case of scalar quarks the above procedure can be repeated and we obtain for the amplitude in position space

$$
G\left(x_{1}, \ldots, x_{M}\right) \equiv\left\langle\prod_{i=1}^{M} \varphi^{\dagger}\left(x_{i}\right) \varphi\left(x_{i}\right)\right\rangle_{\mathrm{conn}}=\sum_{C \ni x_{1}, \ldots, x_{M}} W(C)
$$

or, more explicitly,

$$
\begin{aligned}
& G\left(x_{1}, \ldots, x_{M}\right)=\frac{1}{2^{M}} \int_{0}^{\infty} \mathrm{d} \mathcal{T}\left(\frac{\mathcal{T}}{2 \pi}\right)^{M-1} \mathrm{e}^{-m^{2} \mathcal{T} / 2} \\
& \quad \times \prod_{i=1}^{M-1} \int_{0}^{\phi_{i+1}} \mathrm{~d} \phi_{i} \int_{\substack{z(0)=z(2 \pi)=x_{0} \\
z\left(\phi_{i}\right)=x_{i}}} \mathcal{D} z(\phi) \mathrm{e}^{-\frac{\mu}{2} \int_{0}^{2 \pi} \mathrm{d} \phi \dot{z}^{2}(\phi)} W[z(\phi)],
\end{aligned}
$$


where $\mu=2 \pi / \mathcal{T}$. In momentum space this gives

$$
\begin{aligned}
G\left(\Delta p_{1}, \ldots, \Delta p_{M}\right)=\frac{1}{2^{M}} \int_{0}^{\infty} \mathrm{d} \mathcal{T}\left(\frac{\mathcal{T}}{2 \pi}\right)^{M-1} \mathrm{e}^{-m^{2} \mathcal{T} / 2} \\
\quad \times \prod_{i=1}^{M-1} \int_{0}^{\phi_{i+1}} \mathrm{~d} \phi_{i} \int_{z(0)=z(2 \pi)=0} \mathcal{D} z(\phi) \mathrm{e}^{-\frac{\mu}{2} \int_{0}^{2 \pi} \mathrm{d} \phi \dot{z}^{2}(\phi)+\mathrm{i} \int_{0}^{2 \pi} \mathrm{d} \phi p(\phi) \cdot \dot{z}(\phi)} W[z(\phi)] .
\end{aligned}
$$

In this case there is no $k$-integration and no term involving the gamma matrices. The $\dot{z}^{2}$-term is specific for the scalar case. It is related to the occurrence of the second derivative in the Klein-Gordon operator. Introducing an auxiliary $k$-integration, we can rewrite Eq. (2.23) in the form

$$
\begin{gathered}
G\left(\Delta p_{1}, \ldots, \Delta p_{M}\right)=\frac{1}{2^{M}} \int_{0}^{\infty} \mathrm{d} \mathcal{T}\left(\frac{\mathcal{T}}{2 \pi}\right)^{M-1} \mathrm{e}^{-m^{2} \mathcal{T} / 2} \prod_{i=1}^{M-1} \int_{0}^{\phi_{i+1}} \mathrm{~d} \phi_{i} \int \mathcal{D} k(\phi) \\
\times \int_{z(0)=z(2 \pi)=0} \mathcal{D} z(\phi) \mathrm{e}^{\mathrm{i} \int_{0}^{2 \pi} \mathrm{d} \phi\left[(k(\phi)+p(\phi)) \cdot \dot{z}(\phi)-\mathcal{T} k^{2}(\phi) / 4 \pi\right]} W[z(\phi)]
\end{gathered}
$$

which looks similar to Eq. (2.20).

\section{$3 \quad$ Wilson loop as string disk amplitude}

An old idea is to identify the Wilson loop of large $N$ QCD with the (tree level) disk amplitude in a certain string theory which QCD is equivalent to. As is already pointed out, the simplest Nambu-Goto string in flat space appears to be surprisingly accurate for large loops and reproduces the asymptotic area law (1.3). We shall therefore first review the known results for the bosonic string.

The calculation of the tree level disk amplitude for the Polyakov string has a subtlety associated with fixing conformal gauge $[18,19,20,21]$. The decoupling of the Liouville field $\varphi(r, \sigma)$ is possible only in the interior of the disk, while its boundary value $\varphi(1, \sigma)$ determines the metric at the one-dimensional boundary:

$$
h(\sigma)=\mathrm{e}^{\varphi(1, \sigma) / 2} .
$$

At the classical level this fixes the parametrization of the boundary contour. The path integral over the boundary value of the Liouville field then restores an invariance under reparametrizations of the boundary in quantum case.

\subsection{Unit circle parametrization}

Let us parametrize the unit disk $\mathbb{D}$ by the variables $0<r \leq 1$ and $\sigma \in[0,2 \pi)$. Alternatively, one can map the unit disk onto the upper half-plane:

$$
z=\mathrm{i} \frac{1+r \mathrm{e}^{\mathrm{i} \sigma}}{1-r \mathrm{e}^{\mathrm{i} \sigma}} .
$$


Then the boundary $\partial \mathbb{D}$, associated with $r=1$, is mapped onto the real axis parametrized by $-\infty<s<+\infty$, so that

$$
s(\sigma)=-\cot \frac{\sigma}{2} .
$$

Integrating over the string fluctuations inside the disk, i.e. over $\vec{X}(r, \sigma)$ with $r<1$, we arrive at the boundary action which is a functional of the field

$$
\vec{X}(1, \sigma) \equiv \vec{x}(\sigma)
$$

at the boundary.

Actually the original Fradkin-Tseytlin calculation [22] used the disk parametrization. Their result for the disk amplitude of bosonic string in $d=26$ implies

$$
\Psi[\vec{x}(\sigma)]=\exp \left(-\frac{K}{2} \int_{0}^{2 \pi} \mathrm{d} \sigma_{1} \mathrm{~d} \sigma_{2} \vec{x}\left(\sigma_{1}\right) G^{-1}\left(\sigma_{1}-\sigma_{2}\right) \vec{x}\left(\sigma_{2}\right)\right)
$$

where

$$
K=\frac{1}{2 \pi \alpha^{\prime}}
$$

is the string tension and

$$
G(\sigma)=-\frac{1}{2 \pi} \log (2-2 \cos \sigma)=\frac{1}{\pi} \sum_{m=1}^{\infty} \frac{1}{m} \cos m \sigma
$$

is the corresponding Neumann function for the disk. It obeys

$$
G^{-1}(\sigma)=-\frac{\mathrm{d}^{2}}{\mathrm{~d} \sigma^{2}} G(\sigma)=-\frac{1}{2 \pi(1-\cos \sigma)}=\frac{1}{\pi} \sum_{m=1}^{\infty} m \cos m \sigma .
$$

Using Eq. (3.8) we can rewrite the disk amplitude (3.5) as

$$
\Psi[\vec{x}(\sigma)]=\exp \left(-\frac{K}{2} \int_{0}^{2 \pi} \mathrm{d} \sigma_{1} \mathrm{~d} \sigma_{2} \dot{\vec{x}}\left(\sigma_{1}\right) G\left(\sigma_{1}-\sigma_{2}\right) \dot{\vec{x}}\left(\sigma_{2}\right)\right) .
$$

This formula is pure classical: the exponent is just the classical boundary action while determinants coming from the integration over the fields inside the disk are ignored. For this reason the same result holds for the bosonic part of the classical boundary action of superstring in $d=10$. But more subtle phenomena, such as the Lüscher term, which are due to determinants are not captured by Eq. (3.9).

\subsection{Integration over reparametrizations}

The exponent in Eq. (3.9) is not invariant under reparametrization of the contour:

$$
\vec{x}(\sigma) \rightarrow \vec{x}(\theta(\sigma))
$$


where the reparametrizing function $\theta(\sigma)$ obeys

$$
\theta(0)=0, \quad \theta(2 \pi)=2 \pi, \quad \frac{\mathrm{d} \theta(\sigma)}{\mathrm{d} \sigma} \geq 0 .
$$

Polyakov [23] proposed (see also Ref. [24]) to integrate the disk amplitude (3.9) over the reparametrizations thus providing a reparametrization-invariant disk amplitude which can be identified with the Wilson loop in large $N$ QCD. For a unit-circle parametrization this gives

$$
\Psi[\vec{x}(\cdot)]=\int_{D i f f\left(S^{1}\right)} \mathcal{D}_{\text {diff }} \theta(\sigma) \exp \left(-\frac{K}{2} \int_{0}^{2 \pi} \mathrm{d} \sigma_{1} \mathrm{~d} \sigma_{2} \vec{x}\left(\theta\left(\sigma_{1}\right)\right) G^{-1}\left(\sigma_{1}-\sigma_{2}\right) \vec{x}\left(\theta\left(\sigma_{2}\right)\right) .\right)
$$

or, equivalently,

$$
\Psi[\vec{x}(\cdot)]=\int_{D i f f\left(S^{1}\right)} \mathcal{D}_{\text {diff }} \sigma(\theta) \exp \left(-\frac{K}{2} \int_{0}^{2 \pi} \mathrm{d} \theta_{1} \mathrm{~d} \theta_{2} \dot{\vec{x}}\left(\theta_{1}\right) G\left(\sigma\left(\theta_{1}\right)-\sigma\left(\theta_{2}\right)\right) \dot{\vec{x}}\left(\theta_{2}\right)\right) .
$$

Here the path integration is over the (infinite) group of diffeomorphisms of a circle $\operatorname{Diff}\left(S^{1}\right)$, i.e. over functions with non-negative derivative $\theta^{\prime}(\sigma)$, which obey Eq. (3.11).

An explicit expression for the measure for integrating over reparametrizations can be given using an expansion over the complete set of basis functions $f_{j}(\sigma)$ :

$$
\theta(\sigma)=\sum_{j=1}^{L} \theta_{j} f_{j}(\sigma)
$$

where $f_{j}=1$ at the $j$-th interval $\left[\sigma_{j-1}, \sigma_{j}\right]\left(\sigma_{0}=\sigma_{L}-2 \pi\right)$ with arbitrary $\sigma_{j}$ 's and vanishes otherwise. A continuous function is approached when the number $L$ of intervals becomes infinite and $\sigma_{j}-\sigma_{j-1} \rightarrow 0$. We can then define the measure $\mathcal{D}_{\text {diff }} \theta(\sigma)$ by

$$
\int_{D i f f\left(S^{1}\right)} \mathcal{D}_{\operatorname{diff}} \theta(\sigma) \cdots=\lim _{L \rightarrow \infty} \int_{0}^{2 \pi} \mathrm{d} \theta_{L} \frac{\left(\sigma_{L}-\sigma_{L-1}\right)}{\mid \mathrm{e}^{\mathrm{i} \theta_{L}}-\mathrm{e}^{\mathrm{i} \theta_{L-1} \mid}} \prod_{j=1}^{L-1} \int_{0}^{\theta_{j+1}} \mathrm{~d} \theta_{j} \frac{\left(\sigma_{j}-\sigma_{j-1}\right)}{\mid \mathrm{e}^{\mathrm{i} \theta_{j}}-\mathrm{e}^{\mathrm{i} \theta_{j-1} \mid}} \cdots .
$$

If $\sigma$ is chosen to be the length of an arc of the unit circle, then $\sigma_{j}=2 \pi j / L$. Otherwise, we have $\sigma_{j}=\sigma(2 \pi j / L)$. As distinct from the usual measure $\mathcal{D} \theta(\sigma)$ : the integrals in Eq. (3.15) are ordered, while the additional factors are needed to provide necessary symmetries as is explained in Sect. 4.

If the unit circle is mapped onto the real axis by Eq. (3.3), the disk amplitude takes the form

$$
\Psi[\vec{x}(\cdot)]=\int_{D i f f(\mathbb{R})} \mathcal{D}_{\text {diff }} t(s) \exp \left(-\frac{K}{4 \pi} \int_{-\infty}^{+\infty} \frac{\mathrm{d} s_{1} \mathrm{~d} s_{2}}{\left(s_{1}-s_{2}\right)^{2}}\left[\vec{x}\left(t\left(s_{1}\right)\right)-\vec{x}\left(t\left(s_{2}\right)\right)\right]^{2}\right),
$$


where the path integral over $t(s)$ restores the invariance under reparametrizations

$$
s \rightarrow t(s), \quad t(-\infty)=-\infty, \quad t(+\infty)=+\infty, \quad \frac{\mathrm{d} t}{\mathrm{~d} s} \geq 0 .
$$

The measure on $\operatorname{Diff}(\mathbb{R})$ can be given by

$$
\int_{D i f f(\mathbb{R})} \mathcal{D}_{\text {diff }} t(s) \cdots=\lim _{L \rightarrow \infty} \int_{-\infty}^{+\infty} \mathrm{d} t_{L} \frac{\left(s_{L}-s_{L-1}\right)}{\left(t_{L}-t_{L-1}\right)} \prod_{j=1}^{L-1} \int_{-\infty}^{t_{j+1}} \mathrm{~d} t_{j} \frac{\left(s_{j}-s_{j-1}\right)}{\left(t_{j}-t_{j-1}\right)} \cdots
$$

which is of the same type as in Eq. (3.15).

The equivalence of Eq. (3.16) and Eq. (3.12) can be shown using Eq. (3.3). For the real-axis parametrization the Green function and the inverse one read

$$
G(s)=-\frac{1}{\pi} \log |s|
$$

and

$$
G^{-1}(s)=-\frac{1}{\pi s^{2}} .
$$

These formulas are the counterparts of Eqs. (3.7) and (3.8), while that of Eq. (3.13) is

$$
\Psi[\vec{x}(\cdot)]=\int_{D i f f(\mathbb{R})} \mathcal{D}_{\text {diff }} s(t) \exp \left(\frac{K}{2 \pi} \int_{-\infty}^{+\infty} \mathrm{d} t_{1} \mathrm{~d} t_{2} \dot{\vec{x}}\left(t_{1}\right) \log \left|s\left(t_{1}\right)-s\left(t_{2}\right)\right| \dot{\vec{x}}\left(t_{2}\right)\right) .
$$

To show the equivalence of (3.16) and (3.21) (or (3.12) and (3.13)), we integrate the exponent by parts

$$
\begin{gathered}
\int_{-\infty}^{+\infty} \frac{\mathrm{d} s_{1} \mathrm{~d} s_{2}}{\left(s_{1}-s_{2}\right)^{2}}\left[\vec{x}\left(t\left(s_{1}\right)\right)-\vec{x}\left(t\left(s_{2}\right)\right)\right]^{2}=\int_{-\infty}^{+\infty} \frac{\mathrm{d} t_{1} \mathrm{~d} t_{2} \dot{s}\left(t_{1}\right) \dot{s}\left(t_{2}\right)}{\left[s\left(t_{1}\right)-s\left(t_{2}\right)\right]^{2}}\left[\vec{x}\left(t_{1}\right)-\vec{x}\left(t_{2}\right)\right]^{2} \\
=\frac{1}{2} \int_{-\infty}^{+\infty} \mathrm{d} t_{1} \mathrm{~d} t_{2}\left[\vec{x}\left(t_{1}\right)-\vec{x}\left(t_{2}\right)\right]^{2} \frac{\partial^{2}}{\partial t_{1} \partial t_{2}} \log \left[s\left(t_{1}\right)-s\left(t_{2}\right)\right]^{2} \\
=-\int_{-\infty}^{+\infty} \mathrm{d} t_{1} \mathrm{~d} t_{2} \dot{\vec{x}}\left(t_{1}\right) \dot{\vec{x}}\left(t_{2}\right) \log \left[s\left(t_{1}\right)-s\left(t_{2}\right)\right]^{2}
\end{gathered}
$$

\subsection{Large loops and minimal area}

In spite of the fact that the right-hand side of Eq. (3.16) is derivable, as is already mentioned, for bosonic string in $d=26$ or superstring in $d=10$, where the integration over reparametrizations comes from the integration over the boundary value of the Liouville field, we shall use it only as an ansatz for asymptotically large loops or, equivalently, very 
large $K$, when the integral over reparametrizations has a saddle point at $t(s)=t_{*}(s)$. This will be crucial for reproducing the area-law behavior

$$
\Psi[\vec{x}(\cdot)] \stackrel{\text { large loops }}{\propto} \mathrm{e}^{\left.-K S_{\min }[\vec{x} \cdot \cdot)\right]}
$$

for asymptotically large loops.

The appearance of the area of the minimal surface, spanned by the contour $\vec{x}(\cdot)$, as a minimum of the boundary action in Eq. (3.16) (or Eq. (3.12)) is related to the fact that it is nothing but the functional known in mathematics as the Douglas integral [25], whose minimum with respect to reparametrizations does give the minimal area:

$$
\begin{aligned}
\min _{t(s)} & \left\{\frac{1}{4 \pi} \int_{-\infty}^{+\infty} \mathrm{d} s \int_{-\infty}^{+\infty} \mathrm{d} s^{\prime} \frac{\left[x(t(s))-x\left(t\left(s^{\prime}\right)\right)\right]^{2}}{\left(s-s^{\prime}\right)^{2}}\right\} \\
= & \frac{1}{4 \pi} \int_{-\infty}^{+\infty} \mathrm{d} s \int_{-\infty}^{+\infty} \mathrm{d} s^{\prime} \frac{\left[x\left(t_{*}(s)\right)-x\left(t_{*}\left(s^{\prime}\right)\right)\right]^{2}}{\left(s-s^{\prime}\right)^{2}}=S_{\min }[x(\cdot)] .
\end{aligned}
$$

This issue is clarified in Appendix B.

The necessity of the function $t_{*}(s)$ (or $\theta_{*}(\sigma)$ ), reparametrizing the boundary, is due to the fact that coordinates describing the minimal surface have to obey the condition of the conformal gauge for the quadratic action, used in the Polyakov string formulation, to coincide with the area. In particular, Eq. (3.4) has to be replaced for this reason by

$$
\vec{X}(1, \sigma) \equiv \vec{x}\left(\theta_{*}(\sigma)\right) .
$$

For large loops $\vec{x}(\cdot)$ the path integral over reparametrizations in Eqs. (3.16) or (3.21) has a saddle point, which is denoted above by $\theta_{*}(\sigma)$. The saddle-point value of the boundary action recovers the minimal area $S_{\min }[\vec{x}(\cdot)]$, reproducing the exponential in Eq. (3.23). To say in other words, minimizing the exponential of the Douglas functional over reparametrizations yields the precise area-law

$$
\min _{t(s)}\left\{\mathrm{e}^{-\frac{K}{4 \pi} \int_{-\infty}^{+\infty} \mathrm{d} s \int_{-\infty}^{+\infty} \mathrm{d} s^{\prime} \frac{\left.\left[x(t(s))-x\left(t s^{\prime}\right)\right]\right]^{2}}{\left(s-s^{\prime}\right)^{2}}}\right\}=\mathrm{e}^{-K S_{\min }[x(\cdot)]} .
$$

We shall use this equation in Sect. 5 when calculating scattering amplitudes in QCD.

The Gaussian fluctuations around the saddle-point $\theta_{*}(\sigma)$ result in an appearance of a pre-exponential factor, so Eq. (3.23) is modified as

$$
\Psi[x(\cdot)] \stackrel{\text { large loops }}{=} F[\sqrt{K} x(\cdot)] \mathrm{e}^{-K S_{\min }[x(\cdot)]}\left[1+\mathcal{O}\left(\left(K S_{\min }\right)^{-1}\right)\right],
$$

where the pre-exponential factor $F[\sqrt{K} x(\cdot)]$ is contour dependent. Its calculation for a circle is performed in Appendix C.

Therefore, the asymptotic area law (3.23) is recovered by Eqs. (3.12) [(3.13)] or (3.16) [(3.21)] modulo the pre-exponential which is not essential for large loops. 


\subsection{Remark on the area law for large loops in QCD}

In general, the Wilson loops are not observable in QCD. Observable quantities are scattering amplitudes which are given by the sum over paths of the Wilson loops (see Eq. (2.13)). Nevertheless, some observables can be directly expressed through the Wilson loop of a certain shape. An example is the interaction potential between static quarks which is determined by a $T \times R$ rectangular loop for $T \gg R$.

It is well-known $[26,27,28]$ how this potential is calculable for the Nambu-Goto string, including the Lüscher term. Then the most convenient choice is to parametrize the string world-sheet by the coordinates along the $T$ - and $R$-axes, which are conformal. The results described in the previous subsection allow us to answer a natural question as to how the linear potential can be reproduced for the unit-circle parametrization which does not obey in general the conformal gauge. The reparametrizing function $\theta_{*}(\sigma)$ is determined for the rectangle loop by the Schwarz-Christoffel mapping. Once again, the path integral over reparametrizations in Eq. (3.16) is crucial to identify the asymptote of the string disk amplitude with the asymptotic area-law behavior of the Wilson loop in QCD.

\section{Derivation of the Koba-Nielsen amplitudes}

Scattering amplitudes in open string theory are conventionally obtained by inserting vertex operators in the path integral over string fluctuations. Having the boundary action, they are represented as the path integral over $\vec{x}(\cdot)$ with the vertex operators inserted at the boundary. The disk amplitude plays, therefore, the role of a generating functional for scattering amplitudes.

\subsection{The Fourier transformation}

It is convenient to perform a (functional) Fourier transformation ${ }^{3}$

$$
\Psi[\vec{p}(\cdot)]=\int \mathcal{D} \vec{x} \mathrm{e}^{\mathrm{i} \int \vec{p} \mathrm{~d} \vec{x}} \Psi[\vec{x}(\cdot)],
$$

where the exponent

$$
\int \vec{p} \mathrm{~d} \vec{x}=\int \mathrm{d} t \vec{p} \dot{\vec{x}}
$$

is invariant under reparametrizations. This functional Fourier transformation is of the type as in Eq. (2.20) and transforms the position-space disk amplitude to a momentumspace one.

Substituting (3.16) into Eq. (4.1) and performing the Gaussian integration, we arrive at the following momentum-space disk amplitude

$$
\Psi[\vec{p}(\cdot)]=\int \mathcal{D}_{\text {diff }} t(s) \exp \left(-\frac{\alpha^{\prime}}{2} \int_{-\infty}^{+\infty} \frac{\mathrm{d} s_{1} \mathrm{~d} s_{2}}{\left(s_{1}-s_{2}\right)^{2}}\left[\vec{p}\left(t\left(s_{1}\right)\right)-\vec{p}\left(t\left(s_{2}\right)\right)\right]^{2}\right) .
$$

\footnotetext{
${ }^{3}$ Such a transformation of the Wilson loops was first advocated in Ref. [29].
} 
Note that (4.3) has the same form as (3.16) only with $K$ replaced by $1 / K=2 \pi \alpha^{\prime}$.

In front of the exponential in Eq. (4.3) there is in fact the determinant (in $d=4$ dimensions)

$$
\begin{aligned}
{\left[\operatorname{det}_{t_{1} t_{2}} G\left(s\left(t_{1}\right)-s\left(t_{2}\right)\right)\right]^{-d / 2} } & =\exp \left\{-\frac{d}{2} \int \mathrm{d} t_{1} \log \left[G\left(s\left(t_{1}\right)-s\left(t_{2}=t_{1}\right)\right)\right]\right\} \\
& =\exp \left\{-\frac{d}{2} \int \mathrm{d} t_{1} \log [G(0)]\right\}
\end{aligned}
$$

which is an infinite constant. It can be regularized for the unit-circle parametrization by modifying the Green function (3.7):

$$
G_{b}(\sigma)=-\frac{1}{2 \pi} \log \left(1+b^{2}-2 b \cos \sigma\right)=\frac{1}{\pi} \sum_{m=1}^{\infty} \frac{b^{m}}{m} \cos m \sigma,
$$

which yields

$$
\left[\operatorname{det}_{\theta_{1} \theta_{2}} G\left(\sigma\left(\theta_{1}\right)-\sigma\left(\theta_{2}\right)\right)\right]^{-d / 2}=\exp \left\{-d \pi \log \left[G_{b}(0)\right]\right\}
$$

What is important is that this regularized determinant does not depend on the reparametrizing function $\sigma(\theta)$ and therefore is an overall constant.

The main advantage of the momentum-dependent amplitude (4.3) is that the momentum variable can be chosen to be a step function of $t$ :

$$
\vec{p}(t)=\sum_{j=1}^{M} \vec{p}_{j} f_{j}(t)
$$

where $f_{j}=1$ at the $j$-th interval $\left[t_{j-1}, t_{j}\right]$ and vanishes otherwise as in Eq. (3.14). Note that the stepwise discretization of $\vec{x}(t)$ itself is not possible since it would violate the continuity of the world-line of the string end.

Since $\vec{p}(t)=\vec{p}_{j}$ at the $j$-th interval, the exponential in Eq. (4.3) is in fact a function of $M$ variables $s_{j}: s_{j}<s_{j+1}$. The only effect of the reparametrization (3.17) is then to change the values of $s_{j}$ 's keeping their cyclic order:

$$
\left\{s_{1}<\ldots<s_{j-1}<s_{j}<\ldots<s_{M}\right\} \rightarrow\left\{t_{1}<\ldots<t_{j-1}<t_{j}<\ldots<t_{M}\right\} .
$$

This is a discrete version of the transformation (3.17).

\subsection{From disk amplitude to Koba-Nielsen amplitude}

The stepwise discretization of the momentum-space loop from the previous subsection naturally results in the Koba-Nielsen amplitudes. We present here a simple on-shell derivation which is pretty close to the standard derivations in string theory.

Integrating by parts as in Eq. (3.22), we rewrite the exponent in (4.3) as

$$
\int_{-\infty}^{+\infty} \frac{\mathrm{d} s_{1} \mathrm{~d} s_{2}}{\left(s_{1}-s_{2}\right)^{2}}\left[\vec{p}\left(t\left(s_{1}\right)\right)-\vec{p}\left(t\left(s_{2}\right)\right)\right]^{2}=-\int_{-\infty}^{+\infty} \mathrm{d} t_{1} \mathrm{~d} t_{2} \dot{\vec{p}}\left(t_{1}\right) \dot{\vec{p}}\left(t_{2}\right) \log \left[s\left(t_{1}\right)-s\left(t_{2}\right)\right]^{2} .
$$


For the step functions (4.7), when

$$
\dot{\vec{p}}(t)=-\sum_{j} \Delta \vec{p}_{j} \delta\left(t-t_{j}\right), \quad \Delta \vec{p}_{j} \equiv \vec{p}_{j-1}-\vec{p}_{j}
$$

we find

$$
\Psi\left(\Delta p_{1}, \ldots, \Delta p_{M}\right)=\prod_{i}(1-b)^{\alpha^{\prime} \Delta \vec{p}_{i}^{2}} \int_{s_{1}<\ldots<s_{j-1}<s_{j}<\ldots<s_{M}} \prod_{j} \mathrm{~d} s_{j} \prod_{k \neq l}\left|s_{k}-s_{l}\right|^{\alpha^{\prime} \Delta \vec{p}_{k} \Delta \vec{p}_{l}} .
$$

A few comments concerning Eq. (4.11) are in order. The factor in front of the integral comes from the multipliers with $k=l$. They are made finite by a regularization of the type in Eq. (4.5). The integration over $s_{j}$ 's results from the integration over $s\left(t_{j}\right)$ in the path integral over reparametrizations in Eq. (4.3), while the integration over $s(t)$ at the intermediate points is naively ignored. This integration is in the spirit of the integration over the positions of vertex operators in string theory. Setting $\alpha^{\prime} \Delta \vec{p}_{k}^{2}=1$ (i.e. imposing the tachyonic on-shell condition) and fixing the $P S L(2 ; \mathbb{R})$ invariance, appearing after this

setting, in the standard way, we obtain the on-shell tachyonic amplitude in the KobaNielsen variables.

\subsection{Projective-invariant off-shell amplitudes}

As is well-known, the amplitude (4.11) is invariant under a transformation from the $P S L(2 ; \mathbb{R})$ projective linear group

$$
s \rightarrow s^{\prime}=\frac{a s+b}{c s+d}, \quad a d-b c=1
$$

only when $\alpha^{\prime} \Delta \vec{p}_{k}^{2}=1$, i.e. only for tachyonic amplitudes. On the contrary we might expect that an $M$-particle amplitude, generated by the Fourier transformation of the reparametrization-invariant momentum-space disk amplitude (4.3), should be projectiveinvariant because the projective group is a subgroup of reparametrization transformations.

There are two reasons why (4.3) resulted in Eq. (4.11). The first reason is the divergence of the double integral over $s_{1}$ and $s_{2}$ in the exponent in Eq. (4.3) for $s_{1}=s_{2}$. This integral is of course convergent for smooth $p(t(s))$, but it is divergent for the stepwise $p(t(s))$ when $s_{1}$ and $s_{2}$ lie on adjacent sides $k=l \pm 1$. The correct procedure is to understand this integral according to the principal-value prescription which lead us to the prescription to omit the adjacent sides with $k=l \pm 1$, as is shown in Appendix D.

If we repeat the calculation omitting the sides with $k=l \pm 1$, then the integrations over $s_{1}$ and $s_{2}$ are perfectly finite resulting in

$$
\begin{aligned}
& \sum_{k \neq l \pm 1} \int_{s_{k-1}}^{s_{k}} \mathrm{~d} s_{1} \int_{s_{l-1}}^{s_{l}} \mathrm{~d} s_{2} \frac{\left(p_{k}-p_{l}\right)^{2}}{\left(s_{1}-s_{2}\right)^{2}} \\
& \quad=-2 \sum_{k \neq l} \Delta p_{k} \cdot \Delta p_{l} \log \left|s_{k}-s_{l}\right|-2 \sum_{j} \Delta p_{j}^{2} \log \frac{\left(s_{j}-s_{j-1}\right)\left(s_{j+1}-s_{j}\right)}{\left(s_{j+1}-s_{j-1}\right)}
\end{aligned}
$$


which is projective invariant.

The second reason for the loss of the projective invariance in Eq. (4.11) is that the integration over reparametrizations at intermediate points of a side was not taken into account. A rather subtle issue how to accurately integrate over $s(t)$ at the intermediate points is explained in Appendix D, where it is shown that this integration over reparametrizations at the intermediate points results in the following measure

$$
D^{(M)} s=\prod_{j=1}^{M} \frac{\mathrm{d} s_{j}}{\left|s_{j}-s_{j-1}\right|}
$$

for the integration over $s_{j}$ 's. It is invariant under the projective transformation (4.12) and gives

$$
\begin{aligned}
& \Psi\left(\Delta p_{1}, \ldots, \Delta p_{M}\right) \\
& \quad=\int_{s_{1}<\ldots<s_{i-1}<s_{i}<\ldots<s_{M}} \prod_{i} \frac{\mathrm{d} s_{i}}{\left|s_{i}-s_{i-1}\right|} \prod_{k \neq l}\left|s_{k}-s_{l}\right|^{\alpha^{\prime} \Delta \vec{p}_{k} \Delta \vec{p}_{l}} \prod_{j}\left(\frac{\left(s_{j}-s_{j-1}\right)\left(s_{j+1}-s_{j}\right)}{\left(s_{j+1}-s_{j-1}\right)}\right)^{\alpha^{\prime} \Delta p_{j}^{2}} .
\end{aligned}
$$

For the case of 4 scalars, Eq. (4.15) reproduces the Veneziano amplitude

$$
A\left(\Delta p_{1}, \Delta p_{2}, \Delta p_{3}, \Delta p_{4}\right)=\int_{0}^{1} \mathrm{~d} x x^{-\alpha(s)-1}(1-x)^{-\alpha(t)-1},
$$

where $\alpha(t)=\alpha^{\prime} t$ and

$$
\begin{aligned}
& s=-\left(\Delta p_{1}+\Delta p_{2}\right)^{2}=-\left(\Delta p_{3}+\Delta p_{4}\right)^{2} \\
& t=-\left(\Delta p_{2}+\Delta p_{3}\right)^{2}=-\left(\Delta p_{1}+\Delta p_{4}\right)^{2}
\end{aligned}
$$

are usual Mandelstam's variables (for Euclidean metric). Here the tachyonic condition $\alpha^{\prime} \Delta p_{j}^{2}=1$ has not to be imposed.

\subsection{Ambiguities of the measure}

In Ref. [14] we used, instead of (4.14), another choice of the measure

$$
D^{(M)} s=\prod_{i=1}^{M} \mathrm{~d} s_{i} \frac{\left(s_{i+1}-s_{i-1}\right)}{\left(s_{i}-s_{i-1}\right)\left(s_{i+1}-s_{i}\right)}
$$

which is also invariant under the projective transformation (4.12). This results in the amplitude

$$
\begin{aligned}
& \Psi\left(\Delta p_{1}, \ldots, \Delta p_{M}\right) \\
& =\int_{s_{1}<\ldots<s_{i-1}<s_{i}<\ldots<s_{M}} \prod_{i} \mathrm{~d} s_{i} \prod_{k \neq l}\left|s_{k}-s_{l}\right|^{\alpha^{\prime} \Delta \vec{p}_{k} \Delta \vec{p}_{l}} \prod_{j}\left(\frac{\left(s_{j}-s_{j-1}\right)\left(s_{j+1}-s_{j}\right)}{\left(s_{j+1}-s_{j-1}\right)}\right)^{\alpha^{\prime} \Delta p_{j}^{2}-1} .
\end{aligned}
$$


These formulas are known as the Lovelace choice [30], that reproduces some off-shell dual amplitudes known since late 1960's (for a review see Ref. [31], references therein and subsequent papers $[32,33])$. For the case of 4 scalars, Eq. (4.19) reproduces the Veneziano amplitude (4.16) with $\alpha(t)=1+\alpha^{\prime} t$. It has the same intercept of the Regge trajectory as the on-shell amplitude (4.11) but now the tachyonic condition $\alpha^{\prime} \Delta p_{j}^{2}=1$ has not to be imposed.

The measures (4.14) and (4.18) can be generalized as

$$
D^{(M)} s=\prod_{i} \frac{\mathrm{d} s_{i}}{\left(s_{i}-s_{i-1}\right)}\left(\frac{s_{i+1}-s_{i-1}}{s_{i+1}-s_{i}}\right)^{\alpha_{0}}
$$

where $\alpha_{0}$ is an arbitrary constant. The measures (4.14) and (4.18) are reproduced for $\alpha_{0}=0$ and $\alpha_{0}=1$, respectively. The measure (4.20) is in the spirit of the Koba-Nielsen amplitudes and is projective invariant for an arbitrary $\alpha_{0}$. It is the measure (4.14) which we have obtained in the previous subsection by integrating over reparametrizations, but we cannot exclude that a similar procedure may also exist for other measures.

The amplitude associated with the measure (4.20) is again the Veneziano amplitude (4.16) with $\alpha(t)=\alpha_{0}+\alpha^{\prime} t$. Therefore, only an intercept of the Regge trajectory is sensitive to the choice of the measure provided it is projective invariant. But the change of the measure (4.20) becomes not essential for $1 / \alpha^{\prime} \ll-t \lesssim s$, when the integral over $x$ is dominated by a saddle point.

The Regge trajectory with $\alpha(0) \approx 1$ is usually associated with the vacuum trajectory $=$ Pomeron which appears in QCD from cylinder diagrams $\sim 1 / N^{2}$ (with two quark loops). We work with planar diagrams, single quark loop and, correspondingly, with a quark-antiquark Regge trajectory which has $\alpha(0) \approx 1 / 2$ from experiment.

\section{QCD amplitudes and the area behavior of $\mathrm{W}$}

In the following we ask what is the contribution from the area behavior of the Wilson loop to the corresponding QCD amplitudes. It is of course clear that depending on the kinematical situation there will be other important contributions, e.g. at large transverse momenta where a perturbative behavior of $W$ is relevant.

As discussed in the introduction it is well-known from lattice calculations that the area behavior, and more generally large distance results, have been found to follow the predictions from the Nambu-Goto action rather precisely even at surprisingly low distances. It therefore makes sense to ask what happens to the QCD amplitudes if we approximate the Wilson loop by the area behavior. This question will be studied in the present section.

We now want to insert the Wilson loop given by the area behavior, taking the latter from the celebrated Douglas construction [25] discussed in details in Sect. 3 and Appendix B. We write

$$
W=\mathcal{S} \mathcal{P}_{\sigma} \int \mathcal{D} \sigma(\tau) \exp \left(\frac{K}{4 \pi} \int_{0}^{2 \pi} \mathrm{d} \theta \int_{0}^{2 \pi} \mathrm{d} \theta^{\prime} \dot{z}(\theta) \dot{z}\left(\theta^{\prime}\right) \ln \left(1-\cos \left[\sigma(\theta)-\sigma\left(\theta^{\prime}\right)\right]\right)\right) .
$$


Here $\mathcal{S} \mathcal{P}_{\sigma}$ means that the full integral should be replaced by the Saddle Point with respect to the reparametrizations $\sigma(\phi)$, where $\sigma$ satisfies $\dot{\sigma}>0$ and $\sigma(0)=0$ and $\sigma(2 \pi)=2 \pi$. The integral over $\sigma(\tau)$ would then produce the exponential of the minimal area times a prefactor as displayed in Eq. (3.27). However, since we want to consider QCD, then only the minimal area factor is pertinent, as we know from lattice gauge calculations. In QCD there are subleading factors (perimeter term, Lüscher term, ...), but they are probably not given by the prefactor coming from the saddle point in (5.1). Therefore the $\mathcal{S P}_{\sigma}$ is restricted to include only the leading saddle-point contribution with no multiplicative prefactors.

In the following we want to insert Eq. (5.1) in the basic formula (2.20) with the goal of doing the $z$-integral. If we perform the Douglas saddle point $\mathcal{S P}_{\sigma}$ first, then the resulting minimizing functions $\sigma$ will depend on the curves $C$, and hence also on parameters entering in $z$. As an example, in Appendix B we have discussed the elliptic case, where the minimizing $\sigma$ depends on the ratio of the lengths of the two axes, and these lengths also enter in the expression for $z$. Hence, following this procedure the $z$-integral cannot be performed as a simple Gaussian integral. Therefore, in applying Eq. (2.20) we shall assume that the $\mathcal{S P}_{\sigma}$ operation commutes with the $z$-integral entering in $(2.20)$,

$$
\int \mathcal{D} k(\tau) \int \mathcal{D} z(\tau) \mathcal{S P}_{\sigma} \int \mathcal{D} \sigma(\tau)=\int \mathcal{D} k(\tau) \mathcal{S P}_{\sigma} \int \mathcal{D} \sigma(\tau) \int \mathcal{D} z(\tau)
$$

so that finding the saddle point can wait until the $k$-integral is to be performed. ${ }^{4}$ The validity of Eq. (5.2) is plausible a posteriori, since the $\sigma$-dependence in all situations encountered turns out to occur entirely in the logarithm $\ln \left(1-\cos \left(\sigma(\tau)-\sigma\left(\tau^{\prime}\right)\right)\right)$, as we shall see in the following.

With this assumption we can now perform the Gaussian $z$-integration in the basic formula (2.20) to obtain

$$
\begin{aligned}
& G\left(\Delta p_{1}, \ldots, \Delta p_{M}\right)=\int_{0}^{\infty} \mathrm{d} \mathcal{T} \mathrm{e}^{-m \mathcal{T}} \int_{0}^{\mathcal{T}} \mathrm{d} \tau_{M-1} \prod_{i=1}^{M-2} \int_{0}^{\tau_{i+1}} \mathrm{~d} \tau_{i} \int \mathcal{D} k(\tau) \mathcal{S} \mathcal{P}_{\sigma} \int \mathcal{D} \sigma(\tau) \\
& \times \operatorname{sp~P~} \mathrm{e}^{\frac{\alpha^{\prime}}{2} \int_{0}^{\mathcal{T}} \mathrm{d} \tau \int_{0}^{\mathcal{T}} \mathrm{d} \tau^{\prime}(\dot{k}(\tau)+\dot{p}(\tau)) \cdot\left(\dot{k}\left(\tau^{\prime}\right)+\dot{p}\left(\tau^{\prime}\right)\right) \ln \left(1-\cos \left(\sigma(\tau)-\sigma\left(\tau^{\prime}\right)\right)\right)-\mathrm{i} \int_{0}^{\mathcal{T}} \mathrm{d} \tau \gamma(\tau) \cdot k(\tau)} .
\end{aligned}
$$

Here it should be emphasized that the dots in Eq. (5.1) can be moved to the logarithm by partial integrations, and hence the integration over $z$ can be formulated such that it involves only $z$ itself and not $\dot{z}$. We mention that a similar simplification does not occur in Eq. (2.23) for scalar quarks, due to the occurrence of $\dot{z}^{2}$.

Inserting the stepwise $p(\tau)$, regularizing the integral in the exponent by the principal-

\footnotetext{
${ }^{4}$ It worth noticing that the $\mathcal{S P}_{\sigma}$ operation is in fact nothing but taking the classical limit $\hbar \rightarrow 0$. If the dependence on Planck's constant $\hbar$ is restored, the exponents in both the coordinate-space disk amplitude (3.16) and the momentum-space disk amplitude (4.3) are divided by $\hbar$ because it enters the exponent of the Fourier transformation.
} 
value prescription and using the formula of the type of Eq. (4.13), this becomes

$$
\begin{gathered}
G\left(\Delta p_{1}, \ldots, \Delta p_{M}\right) \propto \prod_{1}^{M-1} \int_{0}^{\phi_{i+1}} \mathrm{~d} \phi_{i} \prod_{j=1}^{M}\left[\frac{\sin \left[\left(\phi_{j+1}-\phi_{j}\right) / 2\right] \sin \left[\left(\phi_{j}-\phi_{j-1}\right) / 2\right]}{\sin \left[\left(\phi_{j+1}-\phi_{j-1}\right) / 2\right] \sin ^{2}\left(\phi_{j} / 2\right)}\right]^{\Delta p_{j}^{2} / 4 \pi K} \\
\times \exp \left(\frac{1}{4 \pi K} \sum_{\substack{i, j=1 \\
i \neq j}}^{M} \Delta p_{i} \Delta p_{j} \ln \left(1-\cos \left(\phi_{i}-\phi_{j}\right)\right)\right) \mathcal{K}\left(\phi_{1}, \ldots, \phi_{M-1} ; \Delta p_{1}, \ldots, \Delta p_{M}\right), \\
\phi_{0}=0, \quad \phi_{M}=2 \pi,
\end{gathered}
$$

where the "kernel" $\mathcal{K}$ is given by

$$
\begin{aligned}
\mathcal{K}= & \int \mathcal{D} k(\theta) \mathcal{S} \mathcal{P}_{\sigma} \int \mathcal{D} \sigma(\phi) \\
& \times \exp \left(\frac{1}{4 \pi K} \int_{0}^{2 \pi} \mathrm{d} \theta \int_{0}^{2 \pi} \mathrm{d} \theta^{\prime} \dot{k}(\theta) \dot{k}\left(\theta^{\prime}\right) \ln \left(1-\cos \left(\sigma(\theta)-\sigma\left(\theta^{\prime}\right)\right)\right)\right) \\
& \times \exp \left(\frac{1}{2 \pi K} \sum_{i} \Delta p_{i} \int_{0}^{2 \pi} \mathrm{d} \theta \dot{k}(\theta) \ln \left(1-\cos \left(\phi_{i}-\sigma(\theta)\right)\right)\right. \\
& \times \int_{0}^{\infty} \mathrm{d} \tau \tau^{M-1} \mathrm{e}^{-m \tau} \operatorname{sp~P} \exp \left(-\frac{\mathrm{i} \tau}{2 \pi} \int_{0}^{2 \pi} \mathrm{d} \phi \gamma(\phi) k(\phi)\right) .
\end{aligned}
$$

Minimizing the kernel $\mathcal{K}$ with respect to $\sigma$ gives the requirement

$$
\int_{0}^{2 \pi} \mathrm{d} \theta^{\prime} \dot{k}(\theta) \dot{k}\left(\theta^{\prime}\right) \cot \frac{\sigma_{*}(\theta)-\sigma_{*}\left(\theta^{\prime}\right)}{2}+2 \sum_{i} \Delta p_{i} \dot{k}(\theta) \cot \frac{\phi_{i}-\sigma_{*}(\theta)}{2}=0 .
$$

For a given $k$ this determines $\sigma_{*}(\theta)$ or, alternatively, the minimizing reparametrization $\theta_{*}(\sigma)$. Using $\sigma_{*}$ it follows by our assumption (5.2) that only the minimal areas are included as contributions to the amplitude.

The main result (5.4) reveals the interesting appearance of poles of the Veneziano type. These occur when two $\phi$ 's coincide, $\phi_{i} \rightarrow \phi_{j}$. The kernel $\mathcal{K}$ does not in general have any pole singularities dependent on the momenta when $\phi_{i} \rightarrow \phi_{j}$, so the momentum dependent Veneziano-type poles cannot be canceled by any contribution from $K$.

The factor

$$
\exp \left(\frac{1}{4 \pi K} \sum_{i \neq j} \Delta p_{i} \Delta p_{j} \ln \left(1-\cos \left(\phi_{i}-\phi_{j}\right)\right)\right)
$$

is independent of the reparametrizations involved in the Douglas construction. This is due to the fact that $\dot{p}$ is a simple sum of delta functions. The above factor is therefore universal. 
After a transformation of the variables $\phi_{i}$, Eq. (5.7) becomes similar to the KobaNielsen representation of the $M$-point function. To see this $u^{5} e^{5}$

$\ln \left[2\left(1-\cos \left(\phi_{i}-\phi_{j}\right)\right)\right]=2 \ln \left|2 \sin \left(\left(\phi_{i}-\phi_{j}\right) / 2\right)\right|=2 \ln \left|s_{i}-s_{j}\right|+\ldots, \quad s_{i}=-\cot \left(\phi_{i} / 2\right)$,

where we left out terms that vanish in the sums occurring in Eq. (5.7) due to energymomentum conservation. The $s_{i}$ 's occur like the variables in the Koba-Nielsen formula. ${ }^{6}$ Thus, in QCD the angular variables, related to the proper-time variables by $\phi_{i}=2 \pi \tau_{i} / \tau$, play the role of the Koba-Nielsen variables.

We thus see that the usual dual model poles are present in the QCD amplitude (5.4). This is indeed to be expected on intuitive grounds, because the area behavior of the Wilson loop can be interpreted in a string framework as arising from the rotating stick with a well-known Regge type spectrum. ${ }^{7}$ It is therefore quite satisfactory that this result also occurs in our general QCD formula when the area law is imposed.

It must be emphasized that in QCD the area law is valid only for large areas, i.e. for large distances. Translating this to momentum space, we need large momenta. Therefore, the low lying dual model spectrum is not relevant in QCD. Thus, the tachyon is also of no relevance, as should be the case.

Going back to Eq. (5.4), it should be noted that this formula does not correspond to a simple dual amplitude, because of the additional factor $\mathcal{K}$. Thus, although the spectrum is quite stringy, the amplitude is more complicated than in the standard dual models. Presumably, this is not too surprising.

As is mentioned above, the tachyon does not occur due to the stringy correspondence large distances=large momenta (in contrast to perturbation theory). Also, when the area behavior leads to extremely small contributions, as is the case for large transverse momenta in string amplitudes (for the 4-point function $-t \sim s$ ), these are not important relative to perturbative contributions ${ }^{8}$, which would then dominate the Wilson loop. This is how the exponential falloff of the 4-particle amplitude with large $-t \sim s$, which is unavoidable in string theory [36], does not happen in our consideration.

Now let us ask if it is possible (at large momenta) to obtain something like a standard dual model. This depends on the extra factor $\mathcal{K}$ in Eq. (5.4). The last factor in the integral over $k$ in (5.5) suggests that we substitute

$$
k \rightarrow k / \tau \text {. }
$$

Then we see that the first two factors in the definition of $\mathcal{K}$ become close to one if $\tau$ is large. For the number of external mesons $M$ large, the $\tau$-integral in Eq. (5.5) is dominated by large

$$
\tau=(M-1) / m
$$

\footnotetext{
${ }^{5}$ Here and below the value of $\phi_{M}$ can be chosen arbitrary, respecting the cyclic symmetry.

${ }^{6}$ The differentials transform as $\mathrm{d} \phi_{i}=2 \mathrm{~d} s_{i} /\left(1+s_{i}^{2}\right)$.

${ }^{7}$ Another derivation of the Regge spectrum from the are law was given in Ref. [34].

${ }^{8}$ We would like to emphasize once again that we are dealing in the large $N$ limit with the quarkantiquark Regge trajectory, whose perturbative QCD calculation was pioneered in Ref. [35].
} 
It is seen from this formula that the values of $\tau$, dominating the $\tau$-integral in Eq. (5.5), are also large for small values of $m$, but we consider such a limit to be rather formal because $m$ has the meaning of a constituent quark mass in QCD, which is about hundred $\mathrm{MeV}$ from experiment even for very small bare masses of up and down quarks because of the spontaneous breaking of chiral symmetry.

When the large values of $\tau$ (5.10) dominate, the integrand of the integral over $\sigma(\phi)$ in Eq. (5.5) does not depend on $\sigma$ and $\mathcal{K}$ degenerates into

$$
\mathcal{K} \propto \int_{\sigma\left(\phi_{i}\right)=\phi_{i}} \mathcal{D} \sigma(\phi)=\prod_{i=1}^{M} \frac{\sin \left(\phi_{i+1} / 2\right) \sin \left(\phi_{i} / 2\right)}{\sin \left[\left(\phi_{i+1}-\phi_{i}\right) / 2\right]}=\prod_{i=1}^{M} \frac{1}{\left|s_{i+1}-s_{i}\right|}
$$

modulo a constant which does not depend on the $\phi_{i}$ 's. The appearance of this factor is due, shortly speaking, to the specifics of the Douglas minimization for stepwise functions $p(\theta(\phi))$ in contrast to smooth functions. The points $\phi_{i}{ }^{\prime} \mathrm{s}$, where the function has discontinuities, are irregular points from the point of view of the minimization because the minimizing function $\theta_{*}(\phi)$ has to satisfy $\theta_{*}\left(\phi_{i}\right)=\phi_{i}$. We can still perform a reparametrization $\phi \rightarrow \theta(\phi)$ at intermediate points $\phi \in\left(\phi_{i}, \phi_{i+1}\right)$. For such a reparametrization $p(\theta(\phi))$ moves along the step but its value remains unchanged. Therefore, this is a zero mode in Douglas' minimization and we have to integrate over these zero modes. For smooth functions, $\theta_{*}(\phi)$ was just fixed. The integration over the zero modes is exactly the same as the integration over reparametrizations at intermediate points described in Subsect. 4.3

and Appendix D. The result of this integration at the interval $\left(\phi_{i}, \phi_{i+1}\right)$ is given by the $i$-th multiplier in Eq. (5.11), while the product runs over the labels of intervals.

The scattering amplitude then takes the form

$$
\begin{aligned}
G\left(\Delta p_{1}, \ldots, \Delta p_{M}\right) \propto & \prod_{i=1}^{M-1} \int_{-\infty}^{s_{i+1}} \frac{\mathrm{d} s_{i}}{1+s_{i}^{2}} \prod_{i=1}^{M} \frac{1}{\left|s_{i+1}-s_{i}\right|}\left[\frac{\left|s_{i+1}-s_{i}\right|\left|s_{i}-s_{i-1}\right|}{\left|s_{i+1}-s_{i-1}\right|}\right]^{\Delta p_{i}^{2} / 2 \pi K} \\
& \quad \times \exp \left(\frac{1}{2 \pi K} \sum_{\substack{i, j=1 \\
i \neq j}}^{M} \Delta p_{i} \Delta p_{j} \ln \left|s_{i}-s_{j}\right|\right)
\end{aligned}
$$

which looks similar to the Koba-Nielsen amplitude. It should be kept in mind that this expression is only valid if the area-law behavior of the Wilson loop dominates over other contributions to $W$, and if the number of external particles $M$ is large and/or $m$ is small compared to $\sqrt{K}$ as is already mentioned.

In Appendix E we have discussed this expression in much more details. In particular we have shown how the Veneziano amplitude with the usual Regge behavior follows.

\section{Large number of external particles WL/SA duality}

The reader may have noted that the area behaved Wilson loop and the large $M$ amplitude before the integrations over the $\phi_{i}$ 's are done look very similar (see Eqs. (5.1) and (5.12)), 
except that the variables $z$ and $p$ are somehow interchanged. This reminds us about the Wilson-loop/scattering-amplitude duality mentioned in the introduction, which was found in SYM perturbation theory, see Refs. [8] and [9].

This similarity can be made explicit by the substitution

$$
z(\phi)=\frac{1}{K} \sum_{i} p_{i} \Theta\left(\phi-\phi_{i}\right) \Theta\left(\phi_{i+1}-\phi\right),
$$

where the $\Theta$ 's are the Heaviside step functions. From this expression we have

$$
\dot{z}(\phi)=\frac{1}{K} \sum_{i} \Delta p_{i} \delta\left(\phi-\phi_{i}\right)=\frac{\dot{\theta}(\phi)}{K} \sum_{i} \Delta p_{i} \delta\left(\theta(\phi)-\theta_{i}\right),
$$

where $\theta=\theta(\phi)$ is some reparametrization with $\theta\left(\phi_{i}\right)=\theta_{i}$. If this equation is inserted in the area behaved Wilson loop (5.1), it reproduces the multiparticle amplitude in (5.12) when integrated over the $\phi_{i}$. The spatial variable on the left-hand side of Eq. (6.1) is on the right-hand side composed of a number of constant vectors given by the momenta, which is rather analogous to the duality (1.2) mentioned in the introduction for the $\mathcal{N}=4$ SYM. A difference is that here we need to integrate over all trajectories considered as functions of $\phi_{i}$.

Equation (6.1) reemphasizes the often mentioned fact that for strings the large $z$ limit is equivalent to the large $p$ limit. The surprising interchange of space and momentum is clearly a stringy effect.

It is easy to check that the expression (6.1) actually satisfies Douglas' variational condition (B.5) or, alternatively, Eq. (B.14). To see this, let us make a variation

$$
\phi(\theta)=\phi_{*}(\theta)+\delta \phi(\theta)
$$

with $\delta \phi$ small and $\phi_{*}$ giving the minimal Douglas functional, as is explained in Appendix B. We have the conditions

$$
z\left(\theta_{*}\left(\phi_{i}\right)\right)=x_{i}
$$

since the curve passes through the points $x_{i}$. Therefore in the variation we need the conditions

$$
\delta \phi\left(\theta_{i}\right)=0
$$

for all $i$. The first variational derivative of the Douglas functional is proportional to

$$
\int_{0}^{2 \pi} \mathrm{d} \theta \int_{0}^{2 \pi} \mathrm{d} \theta^{\prime} \delta \phi(\theta) \dot{z}(\theta) \dot{z}\left(\theta^{\prime}\right) \cot \frac{\phi_{*}(\theta)-\phi_{*}\left(\theta^{\prime}\right)}{2} .
$$

Inserting Eq. (6.2), the expression (6.6) becomes

$$
\sum_{i j} \Delta p_{i} \Delta p_{j} \delta \phi\left(\theta_{i}\right) \cot \frac{\phi_{i}-\phi_{j}}{2}
$$


where we get contributions only from the fixed points $x_{i}$. Because of Eq. (6.5) we see that the first variational derivative (6.7) vanishes. Hence the duality relation (6.1) actually satisfies Douglas' variational principle and in this sense the curves (6.1) represent the dominant trajectories (the "master trajectories") in phase space when the momenta are given. The special trajectory (6.1) is a kind of zero mode solution of the Douglas variational problem, since it does not actually determine the function $\phi_{*}$, which is irrelevant in the case of stepwise constant momenta. We have already discussed this specifics of step functions in Sect. 5 .

To sum up, the result is

$$
\begin{aligned}
G\left(\Delta p_{1}, \ldots, \Delta p_{M}\right) \propto & \prod_{i=1}^{M-1} \int_{0}^{\phi_{i+1}} \mathrm{~d} \phi_{i} \prod_{i=1}^{M} \frac{\sin ^{2}\left(\phi_{i} / 2\right)}{\sin \left[\left(\phi_{i+1}-\phi_{i}\right) / 2\right]} \\
& \times W\left(z(\phi) \rightarrow \frac{1}{K} \sum_{i} p_{i} \Theta\left(\phi-\phi_{i}\right) \Theta\left(\phi_{i+1}-\phi\right)\right),
\end{aligned}
$$

where $W(z(\phi))$ is the Wilson loop as a function of the boundary curve $z(\phi)$. Except for the integrations this is similar to the supersymmetric case (1.2) discussed in [8] and [9].

This similarity can be made even more explicit by noting that Eq. (6.1) means that in the interval $\phi_{i}<\phi<\phi_{i+1}$ the vector $z(\phi)$ is equal to the vector $p_{i} / K$. Remembering that $z$ equals $x_{i}$ and $x_{i+1}$ for the parameter values $\phi_{i}$ and $\phi_{i+1}$, respectively. This amounts to

$$
\Delta p_{i}=K\left(x_{i-1}-x_{i}\right), \quad \text { for } \phi_{i}<\phi<\phi_{i+1},
$$

in conformity with Eq. (1.2).

We again emphasize that all the above results are valid only when the number of external particles $M$ is large. In the general case of a smaller number of produced particles

the situation is much more complex. Presumably there will be important fluctuations around the master trajectory giving contributions to the kernel $\mathcal{K}$.

\section{Conclusions}

We have found a relation between the meson scattering amplitudes and the Wilson loop for large $N$ QCD. We then investigated the behavior of an area behaved $W$ when a functional Fourier transform was performed, leading to the Veneziano multiparticle amplitude in the Koba-Nielsen formulation. This turned out to be very useful when we inserted an area approximation for the Wilson loop in our general expression for the large $N$ QCD amplitude. The result is a convolution integral, with the well-known Koba-Nielsen integrand convoluted with a kernel $\mathcal{K}$. The usual poles always occur because they cannot be prevented by the kernel.

For a very large number of external particles $\mathcal{K}$ becomes essentially a constant, and hence the Veneziano multiparticle amplitude appears. It then turns out that there exists a nice duality between Wilson loops and scattering amplitudes, somewhat similar to the supersymmetric case. 
Although these phenomena are valid for large $N$, one might hope that something similar occurs for $N=3$, in which case this would be observable at LHC, where a huge number of particles are produced. So we hope to see at least some tracks of the Veneziano amplitude in the collider data!

\section{Acknowledgments}

We are indebted to Paolo Di Vecchia, Alexander Gorsky, Alexey Kaidalov, Raffaele Marotta, Niels Obers, and Vladimir Zoller for useful discussions.

\section{Appendix A An example of the use of the "variable" gamma matrix}

Here we shall give an example of how the gamma matrix $\gamma_{\mu}(t)$ operates in the case where we consider the quantity

$$
F=\operatorname{sp} \mathrm{P} \exp \left[\mathrm{i} \int_{0}^{\tau} \mathrm{d} t p_{\mu}(t) \gamma_{\mu}(t)\right]=\operatorname{sp} \prod_{j=n}^{1} \exp \left(\mathrm{i} \not \not_{j}\left(t_{j}-t_{j-1}\right)\right)
$$

where we took the momenta $p$ to be stepwise constant. We can now use

$$
\mathrm{e}^{\mathrm{i} \not \not_{j} \Delta t_{j}}=\cos M \Delta t_{j}+\mathrm{i} \frac{\not p_{j}}{M} \sin M \Delta t_{j}, \quad \Delta t_{j}=t_{j}-t_{j-1}
$$

Here we took $p_{j}^{2}=-M^{2}$ for all $j$, thus assuming that all the external mesons have the same mass. Then

$$
F=\operatorname{sp} \prod_{j=n}^{1}\left(\cos M \Delta t_{j}+\mathrm{i} \frac{\not p_{j}}{M} \sin M \Delta t_{j}\right) .
$$

For the two point case $n=2$ we get

$$
F_{n=2}=4\left(\cos M \Delta t_{1} \cos M \Delta t_{2}-\frac{p_{1} p_{2}}{M^{2}} \sin M \Delta t_{1} \sin M \Delta t_{2}\right) .
$$

Also

$$
F_{n=3}=4\left(c_{1} c_{2} c_{3}-\frac{p_{1} p_{2}}{M^{2}} s_{1} s_{2} c_{1}-\frac{p_{1} p_{3}}{M^{2}} s_{1} c_{2} s_{3}-\frac{p_{2} p_{3}}{M^{2}} c_{1} s_{2} s_{3}\right)
$$

and

$$
\begin{aligned}
F_{n=4}= & 4\left(c_{1} c_{2} c_{3} c_{4}-\frac{p_{3} p_{4}}{M^{2}} c_{1} c_{2} s_{3} s_{4}-\frac{p_{2} p_{4}}{M^{2}} c_{1} s_{2} c_{3} s_{4}-\frac{p_{3} p_{2}}{M^{2}} c_{1} s_{2} s_{3} c_{4}-\frac{p_{1} p_{4}}{M^{2}} s_{1} c_{2} c_{3} s_{4}\right. \\
& -\frac{p_{1} p_{3}}{M^{2}} s_{1} c_{2} s_{3} c_{4}-\frac{p_{1} p_{2}}{M^{2}} s_{1} s_{2} c_{3} c_{4}+\frac{1}{M^{2}}\left(\left(p_{1} p_{2}\right)\left(p_{3} p_{4}\right)-\left(p_{1} p_{3}\right)\left(p_{2} p_{4}\right)\right. \\
& \left.\left.+\left(p_{1} p_{4}\right)\left(p_{2} p_{3}\right)\right) s_{1} s_{2} s_{3} s_{4}\right) .
\end{aligned}
$$

Here $c_{i}=\cos M \Delta t_{i}$ and $s_{i}=\sin M \Delta t_{i}$.

If the momenta are not stepwise constant, we can use the above procedure with $n \rightarrow \infty$, if we divide the interval from 0 to $\tau$ into intervals $\tau / n$ and take the limit at the end. 


\section{Appendix B Douglas' approach to the minimal area}

The Douglas algorithm [25] for finding the area of the minimal surface bounded by a closed contour $C$ which is parametrized by the function $x_{\mu}(\sigma)$ is based on minimizing the boundary functional ${ }^{9}$

$$
A[x(\theta)]=\frac{1}{8 \pi} \int_{0}^{2 \pi} \mathrm{d} \sigma \int_{0}^{2 \pi} \mathrm{d} \sigma^{\prime} \frac{\left[x(\theta(\sigma))-x\left(\theta\left(\sigma^{\prime}\right)\right)\right]^{2}}{1-\cos \left(\sigma-\sigma^{\prime}\right)}
$$

with respect to the reparametrizing functions $\theta(\sigma)(\mathrm{d} \theta(\sigma) / \mathrm{d} \sigma \geq 0)$. The numeric value of $A$ for the given $C$ depends on the choice of $\theta(\sigma)$ and in general

$$
A[x(\theta)] \geq S_{\min }(C)
$$

while the equality is reached for certain function $\theta(\sigma)=\theta_{*}(\sigma)$ which provides the minimum of $A$. The function $\theta_{*}(\sigma)$ is of course contour-dependent.

To prove the fact that

$$
A\left[x\left(\theta_{*}\right)\right]=S_{\min }(C),
$$

we reconstruct $X_{\mu}(r, \sigma)$, describing the surface, in the interior of the unit disk $r<1$ from the boundary value $X_{\mu}(1, \sigma)=x_{\mu}\left(\theta_{*}(\sigma)\right)$ by the Poisson formula and note that thus constructed $X_{\mu}$ automatically obeys conformal gauge

$$
\frac{\partial X}{\partial r} \cdot \frac{\partial X}{\partial \sigma}=0, \quad r^{2} \frac{\partial X}{\partial r} \cdot \frac{\partial X}{\partial r}=\frac{\partial X}{\partial \sigma} \cdot \frac{\partial X}{\partial \sigma} .
$$

Therefore, the Nambu-Goto action coincides for this configuration with the quadratic action and the boundary action in Polyakov string theory coincides with the area.

By varying $A[\theta]$ with respect to $\theta(\sigma)$ at given $C$, we get the following equation for $\theta_{*}(\sigma)$ :

$$
\int_{0}^{2 \pi} \mathrm{d} \sigma^{\prime} \frac{\dot{x}\left(\theta_{*}(\sigma)\right) \cdot\left[x\left(\theta_{*}(\sigma)\right)-x\left(\theta_{*}\left(\sigma^{\prime}\right)\right)\right]}{1-\cos \left(\sigma-\sigma^{\prime}\right)}=0
$$

which can be written in several equivalent forms.

To illustrate how Eq. (B.5) can be used to determine $\theta_{*}(\sigma)$, let us consider the case of a plane contour, when the problem can be solved by a conformal map, and concentrate on the case of an ellipse

$$
x_{1}=a \cos \theta(\sigma), \quad x_{2}=b \sin \theta(\sigma) .
$$

Then Eq. (B.5) takes the form

$$
\int_{0}^{2 \pi} \mathrm{d} \alpha \frac{\sin \left[\theta_{*}(\sigma+\alpha)-\theta_{*}(\sigma)\right]}{1-\cos \alpha}=\epsilon \int_{0}^{2 \pi} \mathrm{d} \alpha \frac{\sin \left[\theta_{*}(\sigma+\alpha)+\theta_{*}(\sigma)\right]-\sin \left[2 \theta_{*}(\sigma)\right]}{1-\cos \alpha}
$$

\footnotetext{
${ }^{9}$ For a modern review see also Ref. [37], Appendix H.
} 
with

$$
\epsilon=\frac{a^{2}-b^{2}}{a^{2}+b^{2}}
$$

For the simplest case of a circle $(a=b)$, the coordinates $r, \sigma$ are conformal so that

$$
\theta_{*}(\sigma)=\sigma \quad \text { circle. }
$$

The right-hand side of Eq. (B.7) vanishes for $\epsilon=0$, while the left-hand side also vanishes for $\theta_{*}(\sigma)$ given by Eq. (B.9).

For $\epsilon \neq 0$ the following ansatz passes through Eq. (B.7):

$$
\theta_{*}(\sigma)=\sigma+\sum_{n \geq 1} c_{n} \sin (2 n \sigma) \quad \text { ellipse, }
$$

after which a set of algebraic equations relating $c_{n}$ 's emerges. For small deforming parameter $\epsilon$, its iterative solution to order $\mathcal{O}\left(\epsilon^{7}\right)$ found by Mathematica is

$$
\begin{aligned}
& c_{1}=\epsilon-\epsilon^{3} / 4+\epsilon^{5} / 8+\mathcal{O}\left(\epsilon^{7}\right) \\
& c_{2}=3 \epsilon^{2} / 4-5 \epsilon^{4} / 8+25 \epsilon^{6} / 64+\mathcal{O}\left(\epsilon^{7}\right) \\
& c_{3}=5 \epsilon^{3} / 6-5 \epsilon^{5} / 4+\mathcal{O}\left(\epsilon^{7}\right) \\
& c_{4}=35 \epsilon^{4} / 32-77 \epsilon^{6} / 32+\mathcal{O}\left(\epsilon^{7}\right) \\
& c_{5}=63 \epsilon^{5} / 40+\mathcal{O}\left(\epsilon^{7}\right) \\
& c_{6}=77 \epsilon^{6} / 32+\mathcal{O}\left(\epsilon^{7}\right) .
\end{aligned}
$$

The minimal area

$$
S_{\min }=\pi a b=\pi a^{2} \sqrt{\frac{1-\epsilon}{1+\epsilon}}
$$

is of course reproduced to this order by substituting (B.10), (B.11) into Eq. (B.1).

Integrating by parts, we can also rewrite the Douglas functional (B.1) as

$$
A=-\frac{1}{4 \pi} \int_{0}^{2 \pi} \mathrm{d} \theta_{1} \int_{0}^{2 \pi} \mathrm{d} \theta_{2} \dot{x}\left(\theta_{1}\right) \cdot \dot{x}\left(\theta_{2}\right) \ln \left(1-\cos \left[\sigma\left(\theta_{1}\right)-\sigma\left(\theta_{2}\right)\right]\right) .
$$

Its variation with respect to $\sigma(\theta)$ results in the equation

$$
\int_{0}^{2 \pi} \mathrm{d} \alpha \dot{x}(\theta) \cdot \dot{x}(\alpha) \cot \left(\frac{\sigma_{*}(\theta)-\sigma_{*}(\alpha)}{2}\right)=0
$$

which is nothing but the Douglas original equation from the Abstract of 1927.

By solving this equation, we obtain the function $\sigma_{*}(\theta)$ which is inverse to $\theta_{*}(\sigma)$, given by Eq. (B.10), and reads

$$
\sigma_{*}(\theta)=\theta+\sum_{n \geq 1} d_{n} \sin (2 n \theta) \quad \text { ellipse }
$$


with

$$
\begin{aligned}
& d_{1}=-\epsilon+\mathcal{O}\left(\epsilon^{7}\right) \\
& d_{2}=\epsilon^{2} / 4+\epsilon^{4} / 8+\epsilon^{6} / 16+\mathcal{O}\left(\epsilon^{7}\right) \\
& d_{3}=-\epsilon^{3} / 12-\epsilon^{5} / 16+\mathcal{O}\left(\epsilon^{7}\right) \\
& d_{4}=\epsilon^{4} / 32+\epsilon^{6} / 32+\mathcal{O}\left(\epsilon^{7}\right) \\
& d_{5}=-\epsilon^{5} / 80+\mathcal{O}\left(\epsilon^{7}\right) \\
& d_{6}=\epsilon^{6} / 192+\mathcal{O}\left(\epsilon^{7}\right) .
\end{aligned}
$$

To proceed further, it is convenient to use a standard ellipse of the area $\pi$ with

$$
a=\sqrt[4]{\frac{1+\epsilon}{1-\epsilon}}, \quad b=\sqrt[4]{\frac{1-\epsilon}{1+\epsilon}} .
$$

Then

$$
\begin{gathered}
x_{1}(\sigma)=a \cos \theta(\sigma)=\sum_{n \geq 1} \mu_{n} \cos (2 n-1) \sigma \\
x_{2}(\sigma)=b \sin \theta(\sigma)=\sum_{n \geq 1} \nu_{n} \sin (2 n-1) \sigma
\end{gathered}
$$

and from Eq. (B.11) $\mu_{n}=\nu_{n}$ to order $\mathcal{O}\left(\epsilon^{7}\right)$.

This can be understood if we continue the boundary coordinates (B.18) inside the unit circle as

$$
\begin{aligned}
& X_{1}(r, \sigma)=\sum_{n \geq 1} \mu_{n} r^{2 n-1} \cos (2 n-1) \sigma \\
& X_{2}(r, \sigma)=\sum_{n \geq 1} \nu_{n} r^{2 n-1} \sin (2 n-1) \sigma .
\end{aligned}
$$

It can be then explicitly verified that these coordinates obeys the conformal gauge (B.4) for $\mu_{n}=\nu_{n}$.

Introducing the analytic function

$$
\mathcal{M}(z)=\sum_{n \geq 1} \mu_{n} z^{2 n-1}
$$

that describes a conformal map of a unit disk onto the interior of the standard ellipse, we get the following algebraic equation

$$
a \cos \theta(\sigma)+\mathrm{i} b \sin \theta(\sigma)=\mathcal{M}\left(\mathrm{e}^{\mathrm{i} \sigma}\right)
$$

or

$$
\mathrm{e}^{\mathrm{i} \theta(\sigma)}=\frac{\mathcal{M}\left(\mathrm{e}^{\mathrm{i} \sigma}\right)+\sqrt{\mathcal{M}^{2}\left(\mathrm{e}^{\mathrm{i} \sigma}\right)-\left(a^{2}-b^{2}\right)}}{a+b}
$$


which determines $\theta(\sigma)$ for the given $\mathcal{M}(z)$. For the ellipse, $\theta$ plays the role of an angular variable in the parametrization (B.6), while the function $\sigma_{*}(\theta)$ relates it to the variable $\sigma$ inherited from the conformal coordinates $r, \sigma$ obeying Eq. (B.4).

An analytic function that describes the conformal map of a unit disk onto the interior of an ellipse was found by Schwarz in 1869 [38] and gives

$$
\mathcal{M}(z)=\sqrt{a^{2}-b^{2}} \sin \left[\frac{\pi}{2 K(s)} F\left(\frac{z}{\sqrt{s}} ; s\right)\right],
$$

where

$$
F(z ; s)=\int_{0}^{z} \frac{\mathrm{d} x}{\sqrt{\left(1-x^{2}\right)\left(1-s^{2} x^{2}\right)}}
$$

and

$$
K(s) \equiv F(1 ; s)=\int_{0}^{1} \frac{\mathrm{d} x}{\sqrt{\left(1-x^{2}\right)\left(1-s^{2} x^{2}\right)}}
$$

are, respectively, the incomplete and complete elliptic integrals of the first kind. ${ }^{10}$ The parameter $s$ is related to $\epsilon$ by

$$
\log \frac{a+b}{a-b}=2 \xi(s) \equiv \frac{\pi K\left(\sqrt{1-s^{2}}\right)}{2 K(s)}
$$

so that iteratively

$$
s=2 \epsilon-\frac{3}{2} \epsilon^{3}+\frac{1}{2} \epsilon^{5}-\frac{1}{32} \epsilon^{7}+\frac{3}{128} \epsilon^{9}+\mathcal{O}\left(\epsilon^{11}\right) .
$$

Substituting the function (B.23) into Eq. (B.21), we obtain the equation

$$
\frac{\pi}{2}-\theta+\mathrm{i} \xi=\frac{\pi}{2 K(s)} F\left(\frac{\mathrm{e}^{\mathrm{i} \sigma}}{\sqrt{s}} ; s\right),
$$

whose imaginary part reproduces Eq. (B.26) in view of the important identity

$$
F\left(\frac{\mathrm{e}^{\mathrm{i} \sigma}}{\sqrt{s}} ; s\right)=F\left(\frac{\mathrm{e}^{-\mathrm{i} \sigma}}{\sqrt{s}} ; s\right)+\mathrm{i} K\left(\sqrt{1-s^{2}}\right) .
$$

The real part of Eq. (B.28) determines the function $\theta(\sigma)$.

In order to obtain it, it is convenient first to rewrite Eq. (B.23) as

$$
\mathcal{M}(z)=\sqrt{a^{2}-b^{2}} \cosh \left[\frac{\pi}{2 K(s)} \int_{0}^{\operatorname{arccosh}(z / \sqrt{s})} \frac{\mathrm{d} \lambda}{\sqrt{1-s^{2} \cosh ^{2} \lambda}}\right] .
$$

\footnotetext{
${ }^{10}$ We use the notations for elliptic integrals from Wikipedia. They are related to those of Mathematica as $F(z ; s)=$ EllipticF $\left[\operatorname{ArcSin}[z], s^{2}\right]$ and $K(s)=\operatorname{EllipticK}\left[s^{2}\right]$.
} 
This form is more suitable for the case when the first argument of $F$ in Eq. (B.23) is large as it is for small $\epsilon$ and correspondingly for small $s$, related by Eq. (B.27). Substituting the function (B.30) into Eq. (B.21), we obtain the equation

$$
\xi+\mathrm{i} \theta=\frac{\pi}{2 K(s)} \int_{0}^{\operatorname{arccosh}\left(\mathrm{e}^{\mathrm{i} \sigma / \sqrt{s})}\right.} \frac{\mathrm{d} \lambda}{\sqrt{1-s^{2} \cosh ^{2} \lambda}}
$$

which is equivalent to Eq. (B.28).

Differentiating Eq. (B.31), we obtain

$$
\theta^{\prime}(\sigma)=\frac{\pi}{2 K(s)} \frac{1}{\sqrt{1+s^{2}-2 s \cos 2 \sigma}}=\frac{\pi}{2 K(s)} \frac{1}{\sqrt{(1-s)^{2}+4 s \sin ^{2} \sigma}}
$$

which yields

$$
\theta(\sigma)=\frac{\pi}{2 K(s)} \int_{0}^{\sigma} \frac{\mathrm{d} \sigma^{\prime}}{\sqrt{(1-s)^{2}+4 s \sin ^{2} \sigma^{\prime}}}=\frac{\pi}{2 K(s)} \frac{1}{(1-s)} F\left(\sin \sigma ; \frac{2 \mathrm{i} \sqrt{s}}{(1-s)}\right)
$$

which is again an elliptic integral of the first kind. This reproduces iteratively Eq. (B.11) provided Eq. (B.27) is satisfied.

The solution (B.33) obeys the properties required for the Douglas minimizing function $\theta_{*}(\sigma)$. It is seen from Eq. (B.32) that $\theta_{*}^{\prime}(\sigma)$ is positive and finite for $s<1$, as is required for a reparametrization. For $s \rightarrow 0$ we have $\theta_{*}^{\prime}(\sigma) \rightarrow 1$ as it should for a circle. In the limit $s \rightarrow 1$ when $K(1)=\infty$ we have $b \rightarrow 0$, so the ellipse collapses. Then $\theta_{*}^{\prime}(\sigma)$ vanishes everywhere except for $\theta=0, \pi$ where it becomes infinite:

$$
\theta_{*}^{\prime}(\sigma) \stackrel{b \rightarrow 0}{\rightarrow} \pi \delta(\sigma)+\pi \delta(\sigma-\pi)
$$

while

$$
\theta_{*}(\sigma) \stackrel{b \rightarrow 0}{\rightarrow} \pi \Theta(\sigma)+\pi \Theta(\sigma-\pi)
$$

is stepwise.

\section{Appendix C The pre-exponential in Eq. (3.27)}

To calculate the pre-exponential in Eq. (3.27), we substitute

$$
\theta(\sigma)=\theta_{*}(\sigma)+\beta(\sigma)
$$

or

$$
\sigma(\theta)=\sigma_{*}(\theta)+\beta(\theta)
$$

where

$$
\beta(0)=\beta(2 \pi)=0
$$


and expand the exponent in Eq. (3.12) (or Eq. (3.13)) to the quadratic order in $\beta$. The linear in $\beta$ term vanishes because $\theta_{*}(\sigma)$ is the extremum while the quadratic part reads

$$
S_{2}[\beta(\theta)]=\frac{K}{2} \int \mathrm{d} \theta_{1} \mathrm{~d} \theta_{2} \dot{\vec{x}}\left(\theta_{1}\right) \dot{\vec{x}}\left(\theta_{2}\right) G^{-1}\left(\sigma_{*}\left(\theta_{1}\right)-\sigma_{*}\left(\theta_{2}\right)\right)\left[\beta\left(\theta_{1}\right) \beta\left(\theta_{2}\right)-\beta^{2}\left(\theta_{1}\right)\right] .
$$

The function $\beta(\theta)$ has to obey

$$
\beta^{\prime}(\theta) \geq-\sigma_{*}^{\prime}(\theta)
$$

for the derivative of the reparametrizing function to be positive. This is always satisfied if $\beta$ is small and smooth enough. Therefore, the measure for the Gaussian path integration over $\beta(\theta)$ is the usual one for smooth functions $\beta(\theta)$ but we shall see subtleties for the functions with large derivative.

In order to calculate the pre-exponential in Eq. (3.27), we need to do the Gaussian integral

$$
I_{2}=\int \mathcal{D} \beta(\theta) \mathrm{e}^{-S_{2}[\beta(\theta)]}
$$

with $S_{2}[\beta(\theta)]$ given by Eq. (C.4).

For a circle of the radius $R$, when

$$
x_{1}(\theta)=R \cos \theta, \quad x_{2}(\theta)=R \sin \theta, \quad x_{3}(\theta)=x_{4}(\theta)=0
$$

and $\sigma_{*}(\theta)=\theta$ according to Eq. (B.9), we have

$$
S_{2}[\beta(\theta)]=-\frac{K R^{2}}{4 \pi} \int_{0}^{2 \pi} \mathrm{d} \theta_{1} \mathrm{~d} \theta_{2} \frac{\cos \left(\theta_{1}-\theta_{2}\right)}{1-\cos \left(\theta_{1}-\theta_{2}\right)}\left[\beta\left(\theta_{1}\right) \beta\left(\theta_{2}\right)-\beta^{2}\left(\theta_{1}\right)\right] .
$$

It is now possible to calculate the path integral over $\beta(\theta)$ by the mode expansion

$$
\beta(\theta)=a_{0}+\sum_{n=1}^{\infty}\left(a_{n} \cos n \theta+b_{n} \sin n \theta\right)
$$

where $a_{0}=-\sum_{i=1}^{\infty} a_{n}$ to obey the boundary condition (C.3) and $a_{n}, b_{n}$ have to satisfy

$$
\sum_{n=1}^{\infty} n\left(-a_{n} \sin n \theta+b_{n} \cos n \theta\right) \geq-1
$$

for the restriction (C.5) to be fulfilled. Inserting Eq. (C.9) into Eq. (C.8), we find

$$
S_{2}=\frac{\pi K R^{2}}{2} \sum_{n=1}^{\infty}(n-1)\left(a_{n}^{2}+b_{n}^{2}\right) .
$$

A consequence of Eq. (C.11) is that $a_{1}$ and $b_{1}$ are zero modes. They are, however, restricted by (C.10) as $-1<a_{1}, b_{1}<1$, so we believe that the integrals over $a_{1}$ and $b_{1}$ simply give a constant. The integrals over nonzero modes are Gaussian and if we were not take into account the restriction (C.10), the result would be

$$
I_{2} \propto \prod_{n=2}^{\infty} \int \mathrm{d} a_{n} \mathrm{~d} b_{n} \mathrm{e}^{-S_{2}}=\prod_{n=2}^{\infty}\left[K R^{2}(n-1) / 2\right]^{-1} .
$$


The infinite product in Eq. (C.12) can be calculated in the standard way, using a regularization via the $\zeta$-function:

$$
\begin{aligned}
& \prod_{n=1}^{\infty} A=A^{\zeta(0)}=A^{-1 / 2} \\
& \prod_{n=1}^{\infty} n=\sqrt{2 \pi} .
\end{aligned}
$$

We then obtain from Eq. (C.12)

$$
I_{2} \propto\left(K R^{2}\right)^{1 / 2}
$$

which fixes the $K R^{2}$ dependence of the pre-exponential in Eq. (3.27).

Since the typical values of $\beta$, which are essential in the path integral over $\beta$ in Eq. (C.6), are $\beta \sim 1 / \sqrt{K} R$, i.e. small for $\sqrt{K} R>>1$, the higher terms of an expansion of $A\left[\theta_{*}(\sigma)+\right.$ $\beta]$ in $\beta$ are suppressed at large $\sqrt{K} R$. The loop expansion goes in the parameter $1 / K R^{2}$ and only one loop contributes with the given accuracy. We thus reproduce the behavior of the type in Eq. (3.27).

We can now ask the question whether or not the typical values of $a_{n}$ 's and $b_{n}$ 's, which are essential in the integral over the nonzero modes in Eq. (C.12), obey the restriction (C.10). We estimate these values as

$$
a_{n} \sim b_{n} \sim\left(n K R^{2}\right)^{-1 / 2}
$$

which are small in accordance with the standard wisdom that high modes are not essential in the Gaussian path integral. However, the restriction (C.10) can be satisfied by a single mode only if

$$
n \lesssim K R^{2}
$$

We assume that this type of the restriction on the number of modes becomes unimportant for $K R^{2} \rightarrow \infty$.

\section{Appendix D Path integrals over $\operatorname{Diff}(\mathbb{R})$}

The measure for integrating over reparametrizations is determined by the metric [21]

$$
\|\delta t\|^{2}=\int_{-\infty}^{+\infty} \mathrm{d} s t^{\prime}(s)[\delta t(s)]^{2}
$$

which is invariant under reparametrizations. It differs from the usual one

$$
\|\delta t\|^{2}=\int_{-\infty}^{+\infty} \mathrm{d} s[\delta t(s)]^{2}
$$

by the presence of $t^{\prime}(s)=\mathrm{d} t(s) / \mathrm{d} s$ in the integrand. 
An explicit representation of the measure $\mathcal{D}_{\text {diff }} t(s)$ for the integration over reparametrizations is given by Eq. (3.18). As distinct from the usual measure $\mathcal{D} t(s)$ :

$$
\int \mathcal{D} t(s) \cdots=\lim _{L \rightarrow \infty} \int_{-\infty}^{+\infty} \prod_{j=1}^{L} \mathrm{~d} t_{j} \cdots
$$

the integrals in Eq. (3.18) are ordered.

The measure (3.15) is the invariant measure on the group Diff $\left(S^{1}\right)$ of reparametrizations (diffeomorphisms) of a circle. Analogously the measure (3.18) is the invariant measure on the group Dif $f(\mathbb{R})$ of reparametrizations (diffeomorphisms) of the real axis. They are defined in the way to be invariant under the $P S L(2 ; \mathbb{R})$ projective transformation at very small but finite discretization spacings $\varepsilon_{i}=s_{i}-s_{i-1} \sim 1 / L$. This guarantees the invariance of the measure under reparametrizations in the limit $L \rightarrow \infty$.

If the integrand of the path integral over reparametrizations is not a functional but a function of, say, only $t\left(s_{j}\right)=t_{j}$ and $t\left(s_{k}\right)=t_{k}$ with $j>k$, we can integrate over intermediate values $t_{i}$ 's with $k<i<j$. Analogously, for a function of $M$ variables we integrate over $t_{i}$ 's inside $M$ intervals. The result of such an integration is a function of the remaining $M$ variables $t_{i_{1}}, \ldots, t_{i_{M}}$, which should be covariant under the projective transformation.

The simplest integral we meet is of the type

$$
\int_{t_{i-1}}^{t_{i+1}} \mathrm{~d} t_{i} \frac{1}{\left(t_{i+1}-t_{i}\right)\left(t_{i}-t_{i-1}\right)}
$$

which is logarithmically divergent at the upper and lower limits of the integration. We regularize it by introducing a small $\delta$ as

$$
\lim _{\delta \rightarrow 0} \int_{t_{i-1}}^{t_{i+1}} \mathrm{~d} t_{i} \frac{\delta}{\left(t_{i+1}-t_{i}\right)^{1-\delta}\left(t_{i}-t_{i-1}\right)^{1-\delta}}=\frac{2}{\left(t_{i+1}-t_{i-1}\right)} .
$$

We can now perform a heuristic derivation of the measure (4.14), which is invariant under the projective transformations, using the following formula

$$
\int_{t_{0}}^{t_{K}} \mathrm{~d} t_{i} \frac{\left[\Gamma\left(\nu_{1}\right)\right]^{-1}}{\left(t_{K}-t_{i}\right)^{1-\nu_{1}}} \frac{\left[\Gamma\left(\nu_{2}\right)\right]^{-1}}{\left(t_{i}-t_{0}\right)^{1-\nu_{2}}}=\frac{\left[\Gamma\left(\nu_{1}+\nu_{2}\right)\right]^{-1}}{\left(t_{K}-t_{0}\right)^{1-\nu_{1}-\nu_{2}}} .
$$

Equation (D.6) is an analogue of the well-known formula

$$
\int_{-\infty}^{+\infty} \frac{\mathrm{d} t_{i}}{\sqrt{2 \pi}} \frac{\mathrm{e}^{-\left(t_{K}-t_{i}\right)^{2} / 2 \nu_{1}}}{\sqrt{\nu_{1}}} \frac{\mathrm{e}^{-\left(t_{i}-t_{0}\right)^{2} / 2 \nu_{2}}}{\sqrt{\nu_{2}}}=\frac{\mathrm{e}^{-\left(t_{K}-t_{0}\right)^{2} / 2\left(\nu_{1}+\nu_{2}\right)}}{\sqrt{\left(\nu_{1}+\nu_{2}\right)}}
$$

which is used for calculations of path integrals with the usual Wiener measure. 
Choosing in Eq. (D.6) $\nu_{1}=\varepsilon_{1} \delta$ and $\nu_{2}=\varepsilon_{2} \delta$ and repeatedly integrating over intermediate points $t_{1}, \ldots, t_{K-1}$, we obtain

$$
\lim _{\delta \rightarrow 0} \prod_{i=1}^{K-1} \int_{t_{0}}^{t_{i+1}} \mathrm{~d} t_{i} \frac{\varepsilon_{i+1} \delta}{\left(t_{i+1}-t_{i}\right)^{1-\varepsilon_{i+1} \delta}} \frac{\varepsilon_{1}}{\left(t_{1}-t_{0}\right)^{1-\varepsilon_{1} \delta}}=\frac{\sum_{i=j}^{M} \varepsilon_{j}}{\left(t_{K}-t_{0}\right)}=\frac{\left(s_{K}-s_{0}\right)}{\left(t_{K}-t_{0}\right)}
$$

Equation (4.14) can be now derived fixing certain $M$ values of $t_{i}$ 's and repeatedly integrating over the intermediate points.

In order to justify Eq. (4.15), we again introduce $L$ infinitesimal intervals $\varepsilon_{i}$ and consider a step function $p(t(s))$ which has discontinuities only at finite number $M$ of points $t_{K_{j}}(j=1, \ldots, M)$, so $\Delta p_{i}=0$ at all other points which we call the intermediate points. To emulate the principal-value integral in the exponent in Eq. (4.3), we omit the terms when $s_{1}$ and $s_{2}$ lie at two adjacent infinitesimal intervals. Similarly to Eq. (4.13) we get

$$
\begin{aligned}
& \sum_{\substack{k, j=1 \\
k \neq l \pm 1}}^{L} \int_{t_{k}}^{t_{k+1}} \mathrm{~d} s_{1} \int_{t_{l}}^{t_{l+1}} \mathrm{~d} s_{2} \frac{\left(p_{k}-p_{l}\right)^{2}}{\left(s_{1}-s_{2}\right)^{2}} \\
& =\lim _{L \rightarrow \infty}\left[-2 \sum_{\substack{j, l=1 \\
j \neq l}}^{L} \Delta p_{j} \cdot \Delta p_{l} \log \left|t_{j}-t_{l}\right|-2 \sum_{j=1}^{L} \Delta p_{j}^{2} \log \frac{\left(t_{j+1}-t_{j-1}\right)\left(t_{j}-t_{j-1}\right)}{\left(t_{j+1}-t_{j-1}\right)}\right] \\
& =-2\left[\sum_{\substack{j, l=1 \\
j \neq l}}^{M} \Delta p_{K_{j}} \cdot \Delta p_{K_{l}} \log \left|t_{K_{j}}-t_{K_{l}}\right|+\sum_{j=1}^{M} \Delta p_{K_{j}}^{2} \log \frac{\left(t_{K_{j+1}}-t_{K_{j-1}}\right)\left(t_{K_{j}}-t_{K_{j-1}}\right)}{\left(t_{K_{j+1}}-t_{K_{j-1}}\right)}\right],
\end{aligned}
$$

where we have substituted $t_{j}$ 's for $s_{j}$ 's and taken into account that $\Delta p_{i}=0$ for the intermediate points with $i \neq K_{j}$.

We still have to insert (D.9) in the exponential and to integrate over the intermediate points. Let us consider a piece from the point $K_{1}$ to the point $K_{3}$ given by the ordered integral of the type

$$
\begin{gathered}
\lim _{\delta \rightarrow 0} \int_{t_{i}<t_{i+1}} \frac{\varepsilon_{K_{1}}}{\left|t_{K_{1}}-t_{K_{1}+1}\right|^{1-\varepsilon_{K_{1}} \delta}} \prod_{i=K_{1}+1}^{K_{2}-1} \mathrm{~d} t_{i} \frac{\varepsilon_{i} \delta}{\left|t_{i}-t_{i+1}\right|^{1-\varepsilon_{i} \delta}}\left[\frac{\left|t_{K_{2}-1}-t_{K_{2}}\right|\left|t_{K_{2}}-t_{K_{2}+1}\right|}{\left|t_{K_{2}-1}-t_{K_{2}+1}\right|}\right]^{\alpha^{\prime} \Delta p_{K_{2}}^{2}} \\
\times \frac{\varepsilon_{K_{2}}}{\left|t_{K_{2}}-t_{K_{2}+1}\right|^{1-\varepsilon_{K_{2}} \delta}} \prod_{i=K_{2}+1}^{K_{3}-1} \mathrm{~d} t_{i} \frac{\varepsilon_{i} \delta}{\left|t_{i}-t_{i+1}\right|^{1-\varepsilon_{i} \delta}} .
\end{gathered}
$$

The integrals over all intermediate points, except for the ones with $i=K_{2}-1$ and $i=K_{2}+1$, are the same as in Eq. (D.8) and are easily doable. The two remaining 
integrals are

$$
\begin{aligned}
= & \left.\lim _{\delta \rightarrow 0} \int_{t_{K_{1}}}^{t_{K_{2}}} \mathrm{~d} t_{K_{2}-1} \int_{t_{K_{2}}}^{t_{K_{3}}} \mathrm{~d} t_{K_{2}+1} \frac{\mathcal{E}_{1} \frac{\varepsilon_{K_{2}-1} \delta}{\left|t_{K_{1}}-t_{K_{2}-1}\right|^{1-\mathcal{E}_{1} \delta}} \frac{\mathcal{E}_{2}}{\left|t_{K_{2}-1}-t_{K_{2}}\right|^{1-\varepsilon_{K_{2}-1} \delta}}}{\left|t_{K_{2}-1}-t_{K_{2}+1}\right|}\right]^{\alpha_{K^{\prime}} p_{K_{2}}^{2}} \frac{\varepsilon_{K_{2}} \delta}{\left|t_{K_{2}}-t_{K_{2}+1}\right|^{1-\varepsilon_{K_{2}} \delta}} \frac{t_{K_{K_{2}+1}-\left.t_{K_{3}}\right|^{1-\mathcal{E}_{1} \delta}}}{}
\end{aligned}
$$

where

$$
\mathcal{E}_{1}=\sum_{i=K_{1}}^{K_{2}-2} \varepsilon_{i}, \quad \mathcal{E}_{2}=\sum_{i=K_{2}+1}^{K_{3}-1} \varepsilon_{i}
$$

For nonvanishing $\Delta p_{K_{2}}^{2}$ the integrals in Eq. (D.11) differ from the one in Eq. (D.8). However, they are also easily calculable for $\delta \rightarrow 0$ when only the domains $\left(t_{K_{3}}-t_{K_{2}+1}\right) \rightarrow 0$ and $\left(t_{K_{2}-1}-t_{K_{1}}\right) \rightarrow 0$ contribute. We get finally

$$
(\mathrm{D} .11) \propto \frac{1}{\left|t_{K_{1}}-t_{K_{2}}\right|}\left[\frac{\left|t_{K_{1}}-t_{K_{2}}\right|\left|t_{K_{2}}-t_{K_{3}}\right|}{\left|t_{K_{1}}-t_{K_{3}}\right|}\right]^{\alpha^{\prime} \Delta p_{K_{2}}^{2}} \frac{1}{\left|t_{K_{2}}-t_{K_{3}}\right|},
$$

thus proving Eq. (4.15).

\section{Appendix E Regge behavior of QCD scattering am- plitude (5.12)}

We show in this appendix that the scattering amplitude (5.12) has asymptotic Regge behavior and reduces to the Veneziano amplitude for $M=4$.

The integrand in Eq. (5.12) is the same as in Eq. (4.15) except for the additional factors $1 /\left(1+s_{i}^{2}\right)$ in the measure. For the 4 -point function we obtain

$$
\begin{aligned}
G_{4}= & {\left[\int_{-\infty}^{s_{4}} \mathrm{~d} s_{3} \int_{-\infty}^{s_{3}} \mathrm{~d} s_{2} \int_{-\infty}^{s_{2}} \mathrm{~d} s_{1}+\int_{-\infty}^{s_{4}} \mathrm{~d} s_{3} \int_{-\infty}^{s_{3}} \mathrm{~d} s_{2} \int_{s_{4}}^{+\infty} \mathrm{d} s_{1}\right.} \\
& \left.+\int_{-\infty}^{s_{4}} \mathrm{~d} s_{3} \int_{s_{4}}^{\infty} \mathrm{d} s_{2} \int_{s_{4}}^{s_{2}} \mathrm{~d} s_{1}+\int_{s_{4}}^{+\infty} \mathrm{d} s_{3} \int_{s_{4}}^{s_{3}} \mathrm{~d} s_{2} \int_{s_{4}}^{s_{2}} \mathrm{~d} s_{1}\right] \\
& \times \frac{1}{\left(1+s_{1}^{2}\right)\left(1+s_{2}^{2}\right)\left(1+s_{3}^{2}\right)} \frac{1}{\left|s_{43}\right|\left|s_{32}\right|\left|s_{21}\right|\left|s_{41}\right|}\left(\frac{s_{21} s_{43}}{s_{31} s_{42}}\right)^{-\alpha^{\prime} s}\left(\frac{s_{41} s_{32}}{s_{31} s_{42}}\right)^{-\alpha^{\prime} t},
\end{aligned}
$$

where we have introduced the notation

$$
s_{i j}=s_{i}-s_{j}
$$


In Eq. (E.1) the integration is only over $s_{1}, s_{2}$ and $s_{3}$, while $s_{4}$ is chosen in a way to preserve the cyclic symmetry but otherwise arbitrary. The difficulty in analyzing the amplitude (E.1) is the presence of the factors $1 /\left(1+s_{i}^{2}\right)$ which violate the $P S L(2 ; \mathbb{R})$ symmetry.

Let us introduce the variable

$$
x=\frac{s_{21} s_{43}}{s_{31} s_{42}}
$$

which runs from 0 at $s_{2}=s_{1}$ to 1 at $s_{2}=s_{3}$ and vise versa. We can use $x$ instead of $s_{2}$ :

$$
s_{2}=s_{4}-\frac{s_{41} s_{43}}{s_{43}+x s_{31}}
$$

and

$$
\frac{\mathrm{d} s_{2}}{\left(1+s_{2}^{2}\right)}=\frac{s_{43} s_{31} s_{41} \mathrm{~d} x}{s_{43}^{2}\left(1+s_{1}^{2}\right)+2 x s_{43} s_{31}\left(1+s_{1} s_{4}\right)+x^{2} s_{13}^{2}\left(1+s_{4}^{2}\right)} .
$$

For the amplitude (E.1) we get

$$
\begin{aligned}
G_{4}= & \int_{0}^{1} \mathrm{~d} x x^{-\alpha^{\prime} s-1}(1-x)^{-\alpha^{\prime} t-1}\left[\int_{-\infty}^{s_{4}} \mathrm{~d} s_{3} \int_{-\infty}^{s_{3}} \mathrm{~d} s_{1}+\int_{-\infty}^{s_{4}} \mathrm{~d} s_{3} \int_{s_{4}}^{+\infty} \mathrm{d} s_{1}+\int_{s_{4}}^{+\infty} \mathrm{d} s_{3} \int_{s_{4}}^{s_{3}} \mathrm{~d} s_{1}\right] \\
& \times \frac{1}{\left(1+s_{1}^{2}\right)\left(1+s_{3}^{2}\right)} \frac{1}{\left|s_{43}\right|\left|s_{31}\right|\left|s_{41}\right|} \frac{\left(s_{43}+x s_{31}\right)^{2}}{\left[s_{43}^{2}\left(1+s_{1}^{2}\right)+2 x s_{43} s_{31}\left(1+s_{1} s_{4}\right)+x^{2} s_{13}^{2}\left(1+s_{4}^{2}\right)\right]},
\end{aligned}
$$

where $s_{4}$ is not necessarily $=\infty$, so the ordering of the points $s_{1}, s_{3}$ and $s_{4}$ preserves cycling symmetry.

The integrand in Eq. (E.6) differs from the projective-covariant one only by the ugly factor

$$
\frac{1}{\left(1+s_{1}^{2}\right)\left(1+s_{3}^{2}\right)} \frac{\left(s_{43}+x s_{31}\right)^{2}}{\left[s_{43}^{2}\left(1+s_{1}^{2}\right)+2 x s_{43} s_{31}\left(1+s_{1} s_{4}\right)+x^{2} s_{13}^{2}\left(1+s_{4}^{2}\right)\right]}
$$

which we shall now see is not important because the integral over $s_{1}$ and $s_{3}$ is divergent.

In order to regularize the integral, we proceed like in Eq. (4.5) changing

$$
\left|s_{i}-s_{j}\right| \rightarrow\left|s_{i}-s_{j}\right|+1-b, \quad b \rightarrow 1
$$

and rescale $s_{i} \rightarrow(1-b) \tilde{s}_{i}$. The linearly divergent part of the integral over $s_{1}$ and $s_{3}$ in Eq. (E.6) decouples from the integral over $x$ and reads

$$
\begin{aligned}
& \frac{1}{1-b}\left[\int_{-\infty}^{\tilde{s}_{4}} d \tilde{s}_{3} \int_{-\infty}^{\tilde{s}_{3}} d \tilde{s}_{1}+\int_{-\infty}^{\tilde{s}_{4}} d \tilde{s}_{3} \int_{\tilde{s}_{4}}^{+\infty} d \tilde{s}_{1}+\int_{\tilde{s}_{4}}^{+\infty} d \tilde{s}_{3} \int_{\tilde{s}_{4}}^{\tilde{s}_{3}} d \tilde{s}_{1}\right] \\
& \times \frac{1}{\left(\left|\tilde{s}_{43}\right|+1\right)\left(\left|\tilde{s}_{31}\right|+1\right)\left(\left|\tilde{s}_{41}\right|+1\right)}+\mathcal{O}\left((1-b)^{0}\right) .
\end{aligned}
$$

This integral is convergent and does not depend on $\tilde{s}_{4}$ because of the invariance under translations.

We have thus reproduced Eq. (4.16), modulo a constant given by Eq. (E.9), with the straight Regge trajectory $\alpha(t)=\alpha^{\prime} t$. The Regge behavior comes about in the same way as when there is the $\operatorname{PSL}(2 ; \mathbb{R})$ symmetry. 


\section{References}

[1] H. B. Nielsen and P. Olesen, A parton view on dual amplitudes, Phys. Lett. B 32 (1970) 203;

B. Sakita and M. A. Virasoro, Dynamical model of dual amplitudes, Phys. Rev. Lett. 24 (1970) 1146.

[2] G. 't Hooft, A planar diagram theory for strong interactions, Nucl. Phys. B 72 (1974) 461.

[3] Y. Makeenko, Methods of contemporary gauge theory, Cambridge Univ. Press (2002).

[4] J. Maldacena, The large $N$ limit of super-conformal field theories and supergravity, Adv. Theor. Math. Phys. 2 (1998) 231 [arXiv:hep-th/9711200].

[5] S. S. Gubser, I. R. Klebanov, and A. M. Polyakov, Gauge theory correlators from non-critical string theory, Phys. Lett. B 428 (1998) 105 [arXiv:hep-th/9802109];

E. Witten, Anti-de Sitter space and holography, Adv. Theor. Math. Phys. 2 (1998) 253 [arXiv:hep-th/9802150].

[6] O. Aharony, S. S. Gubser, J. Maldacena, H. Ooguri, and Y. Oz, Large $N$ field theories, string theory and gravity, Phys. Rep. 323 (2000) 183 [arXiv:hep-th/9905111].

[7] Z. Bern, L. J. Dixon, and V. A. Smirnov, Iteration of planar amplitudes in maximally supersymmetric Yang-Mills theory at three loops and beyond, Phys. Rev. D 72 (2005) 085001 [arXiv:hep-th/0505205].

[8] L. F. Alday and J. Maldacena, Gluon scattering amplitudes at strong coupling, JHEP 0706 (2007) 064 [arXiv:0705.0303 [hep-th]].

[9] J. M. Drummond, G. P. Korchemsky, and E. Sokatchev, Conformal properties of four-gluon planar amplitudes and Wilson loops, Nucl. Phys. B 795 (2008) 385;

A. Brandhuber, P. Heslop, and G. Travaglini, MHV Amplitudes in N=4 super YangMills and Wilson loops, Nucl. Phys. B 794 (2008) 231 [arXiv:0707.1153 [hep-th]]; J. M. Drummond, J. Henn, G. P. Korchemsky, and E. Sokatchev, On planar gluon amplitudes/Wilson loops duality, Nucl. Phys. B 795 (2008) 52 [arXiv:0709.2368 [hepth]].

[10] L. F. Alday and R. Roiban, Scattering amplitudes, Wilson loops and the string/gauge theory correspondence, Phys. Rep. 468 (2008) 153 [arXiv:0807.1889 [hep-th]].

[11] M. Lüscher and P. Weisz, Quark confinement and the bosonic string, JHEP 0207 (2002) 049 [arXiv:hep-lat/0207003].

[12] A. Athenodorou, B. Bringoltz, and M. Teper, The closed string spectrum of $S U(N)$ gauge theories in $2+1$ dimensions, Phys. Lett. B 656 (2007) 132 [arXiv:0709.0693 [hep-lat]];

R. Narayanan and H. Neuberger, Universality of large $N$ phase transitions in Wilson loop operators in two and three dimensions, JHEP 0712 (2007) 066 [arXiv:0711.4551 [hep-th]].

[13] P. Olesen, Strings and QCD, Phys. Lett. B 160 (1985) 144. 
[14] Y. Makeenko and P. Olesen, Implementation of the duality between Wilson loops and scattering amplitudes in QCD, Phys. Rev. Lett. 102 (2009) 071602 [arXiv:0810.4778 [hep-th]].

[15] K. G. Wilson, Confinement of quarks, Phys. Rev. D 10 (1974) 2445.

[16] Y. M. Makeenko and A. A. Migdal, Quantum chromodynamics as dynamics of loops, Nucl. Phys. B 188 (1981) 269.

[17] A. A. Migdal, Loop equations and 1/N expansion, Phys. Rep. 102 (1983) 199.

[18] O. Alvarez, Theory of strings with boundaries: fluctuations, topology and quantum geometry, Nucl. Phys. B 216 (1983) 125.

[19] B. Durhuus, P. Olesen, and J.L. Petersen, On the static potential in Polyakov's theory of the quantized string, Nucl. Phys. B 232 (1984) 291.

[20] A. G. Cohen, G. W. Moore, P. C. Nelson, and J. Polchinski, An off-shell propagator for string theory, Nucl. Phys. B 267 (1986) 143.

[21] A. M. Polyakov, Gauge fields and strings, Harwood Acad. Pub. (1987).

[22] E. S. Fradkin and A. A. Tseytlin, Nonlinear electrodynamics from quantized strings, Phys. Lett. B 163 (1985) 123.

[23] A. M. Polyakov, Talk at the Workshop "Particles, Fields and Strings", Vancouver, July 1997, unpublished.

[24] V. S. Rychkov, Wilson loops, D-branes, and reparametrization path integrals, JHEP 0212 (2002) 068 [arXiv:hep-th/0204250].

[25] J. Douglas, Solution of the problem of Plateau, Trans. Amer. Math. Soc. 33 (1931) 263.

[26] O. Alvarez, Static potential in string theory, Phys. Rev. D 24 (1981) 440.

[27] J. F. Arvis, The exact $\bar{q} q$ potential in Nambu string theory, Phys. Lett. B 127 (1983) 106.

[28] P. Orland, Evolution of fixed-end strings and the off-shell disk amplitude, Nucl. Phys. B 605 (2001) 64 [arXiv:hep-th/0101173].

[29] A. A. Migdal, Momentum loop dynamics and random surfaces in $Q C D$, Nucl. Phys. B 265 (1986) 594.

[30] C. Lovelace, Simple N-Reggeon vertex, Phys. Lett. B 32 (1970) 490.

[31] P. Di Vecchia, Multiloop amplitudes in string theories, in "String Quantum Gravity and Physics at the Planck Energy Scale, Erice 1992", ed. N. Sanchez, World Scientific (1993) p. 16.

[32] L. Cappiello, A. Liccardo, R. Pettorino, F. Pezzella, and R. Marotta, Prescriptions for off-shell bosonic string amplitudes, Lect. Notes Phys. 525 (1999) 466 [arXiv:hepth/9812152].

[33] A. Liccardo, F. Pezzella, and R. Marotta, Consistent off-shell tree string amplitudes, Mod. Phys. Lett. A 14 (1999) 799 [arXiv:hep-th/9903027]. 
[34] A. Y. Dubin, A. B. Kaidalov, and Yu. A. Simonov, Dynamical regimes of the QCD string with quarks, Phys. Lett. B 323 (1994) 41;

Yu. A. Simonov, Spectrum and Regge-trajectories in QCD, Phys. Atom. Nucl. 66 (2003) 2038 [arXiv:hep-ph/0210309].

[35] R. Kirschner and L. N. Lipatov, Double logarithmic asymptotics of quark scattering amplitudes with flavor exchange, Phys. Rev. D 26 (1982) 1202; Double logarithmic asymptotics and Regge singularities of quark amplitudes with flavor exchange, Nucl. Phys. B 213 (1983) 122.

[36] D. J. Gross and P. F. Mende, The High-energy behavior of string scattering amplitudes, Phys. Lett. B 197 (1987) 129.

[37] A. A. Migdal, Loop equation and area law in turbulence, Int. J. Mod. Phys. A 9 (1994) 1197 [arXiv:hep-th/9310088].

[38] Z. Nehari, Conformal mappings, McGraw-Hill, New York (1952). 